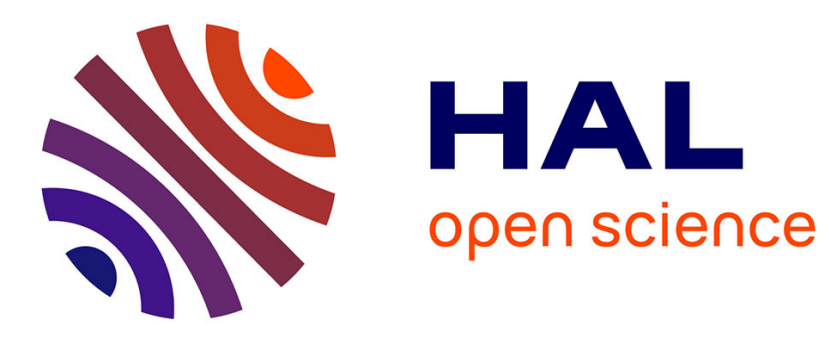

\title{
Bone orthotropic remodeling as a thermodynamically-driven evolution
}

M. Martin, T. Lemaire, Guillaume Haiat, P Pivonka, V. Sansalone

\section{To cite this version:}

M. Martin, T. Lemaire, Guillaume Haiat, P Pivonka, V. Sansalone. Bone orthotropic remodeling as a thermodynamically-driven evolution. Journal of Mechanics in Medicine and Biology, 2020, 20 (4), pp.1950084. hal-02343624

\section{HAL Id: hal-02343624 \\ https://hal.science/hal-02343624}

Submitted on 3 Nov 2019

HAL is a multi-disciplinary open access archive for the deposit and dissemination of scientific research documents, whether they are published or not. The documents may come from teaching and research institutions in France or abroad, or from public or private research centers.
L'archive ouverte pluridisciplinaire HAL, est destinée au dépôt et à la diffusion de documents scientifiques de niveau recherche, publiés ou non, émanant des établissements d'enseignement et de recherche français ou étrangers, des laboratoires publics ou privés. 


\title{
Bone orthotropic remodeling as a thermodynamically-driven evolution
}

\section{MARTIN ${ }^{1,2}$, T. LEMAIRE $^{1}$, G. HAIAT ${ }^{1}$, P. PIVONKA ${ }^{2}$, and V. SANSALONE ${ }^{1, *}$}
${ }^{1}$ Laboratoire Modélisation et Simulation Multi Echelle, MSME UMR 8208 CNRS, Université Paris-Est Créteil, 61 avenue du
Général de Gaulle, 94010 Créteil Cedex, France
${ }^{2}$ Biomechanics and Spine Research Group, School of Chemistry, Physics and Mechanical Engineering, Queensland University of Technology, 2 George St, Brisbane City QLD 4000, Australia *email: vittorio.sansalone@u-pec.fr

November 2, 2019

\begin{abstract}
In this contribution we present and discuss a model of bone remodeling set up in the framework of the theory of generalized continuum mechanics and first introduced by DiCarlo et al. [1]. Bone is described as an orthotropic body experiencing remodeling as a rotation of its microstructure. Thus, the complete kinematic description of a material point is provided by its position in space and a rotation tensor describing the orientation of its microstructure. Material motion is driven by energetic considerations, namely by the application of the Clausius-Duhem inequality to the microstructured material. Within this framework of orthotropic remodeling, some key features of the remodeling equilibrium configurations are deduced in the case of homogeneous strain or stress loading conditions. First, it is shown that remodeling equilibrium configurations correspond to energy extrema. Second, stability of the remodeling equilibrium configurations is assessed in terms of the local convexity of the strain and complementary energy functionals hence recovering some classical energy theorems. Eventually, it is shown that the remodeling equilibrium configurations are not only highly dependent on the loading conditions, but also on the material properties.
\end{abstract}

\section{Introduction}

The present work focuses on the representation of bone adaptation as an evolution of the material principal directions. Bone is a living material constantly 
undergoing microstructural changes. Since the theory of the mechanostat by Frost [2], it has been widely accepted that the evolution of bone mass and structure can be seen as a feedback from the mechanical environment. In particular, the early work of von Meyer[3] and Wolff[4] on the one hand and of Roux[5] on the other hand underline the anisotropic pattern of bone architecture.

In 1867, benefiting from his collaboration with the German civil engineer Culmann, Von Meyer notes a striking connection between the stress lines in a crane and the trabecular architecture in a femur[3,6]. A few years later, Wolff introduces a new idea about bone remodeling, known as Wolff's law, stating that the mechanical function of bone drives the evolution of its architecture[4]. The work of Wolff[7, 8] inspired a wealth of experimental studies investigating the heterogeneous distribution of mass in bone and the pattern of bone architecture[9, 10, 11, 12, 13].

Towards the end of the 20th century, the pioneering work of Carter and Cowin opens the way to two modeling approaches to bone remodeling aiming at explaining the trabecular pattern of bone-considered as evidence of Wolff's lawin terms of mechanical stimuli. On the one hand, Carter and coworkers[14] interpret the trabecular pattern in terms of the heterogeneous distribution of bone mass density. Initially proposed for an isotropic material, this approach is later extended to account for anisotropic remodeling[15]. On the other hand, Cowin introduces the fabric tensor to translate the orthotropic arrangement of the microstructure of a porous material to the continuum level[16, 17]. Since the first works of Carter and Cowin, several studies have been aiming to address how the connection between mechanical loading and bone remodeling can explain bone anisotropy. Some of them investigate the change in trabecular anisotropic architecture using Finite Element simulations at the microstructural scale $(\mu \mathrm{FE})$ to assess bone apposition and resorption. The majority of these micro-scale models rely on the well-documented experimental evidence of a connection between bone remodeling and mechanical loading $[18,19,20]$ and introduce remodeling laws driven by either the strain energy[18, 21, 22, 23, 24, 25, 26, 27], the strain[19] or the stress[28]. Other voxel-based models include uncertainty in the remodeling process, with[21, 22] or without mechanical feedback[29, 30].

Besides these micro-scale models, other works attempt to describe bone orthotropic remodeling at a higher scale. Among the studies dealing with anisotropy at the tissue scale, the majority of works are concerned with characterizing the final stage of bone adaptation which is intended as an optimal state of the trabecular architecture. In 1986, Cowin[17] introduces a mathematical condition for the remodeling equilibrium of bone trabecular architecture. He argues that, according to Wolff's law, the principal axes of the bone fabric tensor-encoding the orientation of bone microstructure-and of the stress tensor must coincide at the remodeling equilibrium. Moreover, he demonstrates that these directions also correspond to the principal axes of the strain tensor. A few years later, several studies focused on material orthotropic optimization. In all generality with respect to an orthotropic material, Rovati et Taliercio[31] and, later on, Banichuk[32], study material orthotropic optimization in relation with the variations of the strain energy, showing that energy extrema always 
correspond to the alignment of the material axes with principal strains. In 1989 , Pedersen performs a 2D study showing that the strain energy density is always stationary for a configuration where material axes and strain principal axes are aligned[33]. Pedersen, joined by Cowin[34, 35], underlines that the nature of the optimum depends on the elastic moduli and in particular on the shear stiffness[33]. Additionally, Cowin demonstrates that, unlike for cubic symmetries, the optimization of strain energy not only depends on orientation but also on the loading configuration. Several authors also apply the concept of bone structural optimization to compare it with known trabecular patterns[36, 37]: the optimization relies on the minimization of an energy-related cost function, while guaranteeing balance equations.

Other studies address bone anisotropic remodeling as a continuous evolution of the tissue-scale material properties. Several of them find the anisotropic pattern as a consequence of the inhomogeneity of the mass density gradient $[14,15$, $38,39]$. Others use a multi-linear law-in line with the ideas of Huiskes[40]-to quantify the rotation of the material axes[41] or the evolution of both elastic moduli (possibly derived from the bone density) and material axes[42, 43], or design a polynomial law for the time rates of the density and fabric tensor[44]. To the authors' knowledge, Jacobs et alare the pioneers in the development of a remodeling law for an anisotropic material[15]. They use the instantaneous dissipation rate, i.e. the difference between the power associated with the external loads and the rate-of-change of the internal energy, as a measure of the effectiveness of the adaptive response and eventually derive the expression of the rate-of-change of the elastic tensor by solving a constrained optimization problem. The notion of dissipation plays also a central role-yet different than in Jacobs' work-in other modeling approaches drawing on the theory of continuum thermodynamics[45, 46, 47, 48, 49, 50]. In particular, DiCarlo and coworkers[1] developed a novel, thermodynamically-consistent theory to describe bone orthotropic remodeling. Instead of devising a remodeling criterion, they derived the evolution law of the material axes from the dissipation principle through a generalized continuum formulation without any ad hoc assumption.

All the above models provide a phenomenological description of bone remodeling since they connect mechanical stimuli and bone response disregarding the underlying biological phenomena. In the last decades, stepping from the pioneering work of Roux[5], a wealth of work at the interface of engineering and biology paved the way to a new class of mechanobiological models of bone. These approaches, bridging a mathematical modeling of cell dynamics with a continuum description of bone, have the potential to provide a much deeper insight on the complex interactions between mechanics and biochemistry that trigger bone cell activity and, in turn, bone remodeling $[51,52,53,54,55,56]$. Mechanobiological models can effectively contribute to understand the biological basis of bone remodeling and have progressively displaced the focus of the bone community from the structure to the process-that is from Wolff's to Roux' point of view. Nevertheless, phenomenological models are still useful, especially in those situations where the focus is set on the mechanical stimuli (e.g. for a better design of prostheses[40]) since they allow a straightforward view of the 
long term macroscopic influence of changes in the external stress field on the overall trend of bone material properties.

In the present paper, we use the theoretical framework proposed by DiCarlo and coworkers[1] and describe bone remodeling as a stress-driven process set up in the framework of generalized continuum mechanics and thermodynamics. As described in the early works of Germain[57], generalized continuum mechanics allows the description of the complex behavior of materials with microstructure. In this framework, the classical Cauchy continuum model is enriched with additional parameters which are meant to provide an average description of the state of the underlying microstructure at the continuum scale. In the case of bone remodeling, these additional parameters may be related to bone micro-architecture as well as its biological and chemical composition. While the work of DiCarlo[1] focuses on the evolution of the material axes, the framework that they developed lies within the general concept of material evolution stemming from the generalized continuum mechanics. In the present work, we keep that framework and evaluate the consistency of our formulation through several theoretical and numerical considerations. Hence, we study here the relationship between the variations of the strain energy through remodeling and the stress and strain state, as well as the material properties. Our main results concern some sound energetic conditions characterizing the remodeling equilibrium. Moreover, we perform a numerical study showing in which cases the alignment of the fabric- with principal stress- and strain directions inferred by Cowin[17] is verified and in which cases it is not.

\section{Modeling bone as a continuum with rotating microstructure}

The theory of material remodeling proposed by DiCarlo and co-workers in the early 2000s[47] paved the way for the general idea to describe the adaptive behavior of living materials in a thermodynamically consistent framework. Functional adaptation of living materials - emerging as the appearance of residual stress or changes in material properties-is described in this theory as the timeevolution of suitable kinematic parameters describing the material state at the continuum level. Evolution laws of these parameters are obtained through a generalized statement of the virtual power principle and thermodynamically consistent constitutive assumptions.

This general theory was used by DiCarlo et al[1] to describe bone remodeling as a rotation of bone material principle directions. In general, bone remodeling results from the change of both density and microarchitecture of the bone material triggered by mechanical and biochemical stimuli. In terms of elasticity, this phenomenon emerges as an evolution of the elastic moduli and principle directions. Following DiCarlo et al[1], in this paper we are only concerned with this latter feature and leave the development of the general model to future work. As long as the elastic moduli do not change, bone remodeling solely emerges 


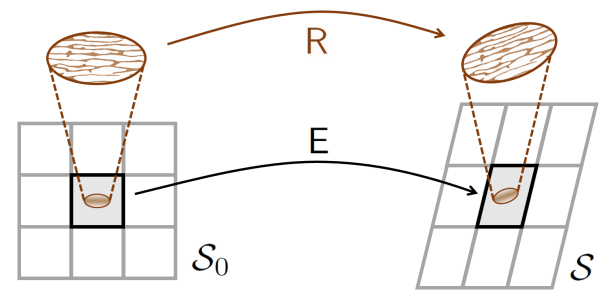

Figure 1: Kinematic description of a body undergoing deformation and rotary remodeling. The visible deformation from the reference shape $\mathcal{S}_{0}$ to the actual shape $\mathcal{S}$ is described by the small strain tensor $\mathrm{E}$ and the rotation of the microstructure is described by the rotation tensor R.

from the rotation of the elasticity axes and will be called rotary remodeling. Assuming bone to be an orthotropic material, remodeling will thus depict the rotation of the orthotropy material axes. In the following subsection we will outline the kinematics, balance and constitutive theory introduced by DiCarlo et al $[1]$.

\section{$2.1 \quad$ Kinematics}

We model a piece of bone as a body $\mathcal{B}$-a continuum collection of material points $b$-occupying at each time $t$ of the time-line $\mathcal{T}$ a closed region of the 3 -dimensional (3D) Euclidean manifold $\mathcal{E}$. Thereafter, the dependency of the different fields on space and time is left understood where unnecessary.

In our framework, the evolution of $\mathcal{B}$ from a reference shape $\mathcal{S}_{0} \in \mathcal{E}$ is thoroughly described by two kinematic fields related to its shape and texture. On the one hand, its visible shape $\mathcal{S} \in \mathcal{E}$ is described by the gross (i.e. coarse grain) displacement field $\mathbf{u}$. On the other hand, the textural change is depicted by a rotation tensor field $\mathrm{R}$ describing the orientation of the microstructureto be called micro-orientation for short-with respect to a fixed frame. Thus, the tensor $\mathrm{R}$ bears quite the same meaning as the fabric tensor introduced by Cowin[17]. Note that, since the strain experienced by bone tissue is small (it does not exceed $1 \%$ in vivo [58]), the small strain tensor $\mathrm{E}$ (i.e., the symmetric part of the displacement gradient) can be used to describe the gross deformation of the body. However, the rotation tensor R does not need to be small. Fig. 1 depicts the basic kinematic ingredients of our model.

In view of the above assumptions, the complete motion of $\mathcal{B}$ is described through the gross and remodeling velocity fields $\mathbf{v}:=\dot{\mathbf{u}}$ and $W:=\dot{R} R^{\top}$, respectively, where a superposed dot denotes time differentiation and superscript "T" denotes transposition. Note that $\mathbf{u}$ and $\mathbf{v}$ take values in $\mathbf{V}$ - the vector spaces of the translations of $\mathcal{E}, \mathrm{R}$ in $\mathrm{Orth}^{+}$- the vector space of the 2nd-order rotation tensors, and W in Skw - the vector spaces of the skew-symmetric 2nd-order tensors. 


\subsection{Balance}

The balance laws governing the evolution of $\mathcal{B}$ are obtained through a generalized statement of the principle of virtual power. To this aim, test velocities related to the gross and remodeling velocities are introduced and the following expressions of the powers of internal and external forces are postulated:

$$
\begin{array}{r}
P_{i}(\hat{\mathbf{v}}, \hat{\mathrm{W}})=\int_{\mathcal{B}}\left(\mathbf{b}_{i} \cdot \hat{\mathbf{v}}-\mathrm{S}: \nabla \hat{\mathbf{v}}+\stackrel{\mathrm{T}}{\mathrm{T}}: \hat{\mathrm{W}}\right), \\
P_{e}(\hat{\mathbf{v}}, \hat{\mathrm{W}})=\int_{\mathcal{B}}(\mathbf{b} \cdot \hat{\mathbf{v}}+\stackrel{\mathrm{T}}{\mathrm{T}}: \hat{\mathrm{W}})+\int_{\partial \mathcal{B}} \mathbf{t}_{\partial} \cdot \hat{\mathbf{v}} .
\end{array}
$$

In the above expressions, integrals are taken either on the body $\mathcal{B}$ or on its boundary $\partial \mathcal{B}$. Moreover, $\hat{\mathbf{v}}$ stands for the $\mathbf{V}$-valued virtual gross velocity and $\hat{W}$ for the Skw-valued virtual micro-rotation velocity. Additionally, $\mathbf{b}_{i}$ and $\mathbf{b}$ are the inner and outer bulk forces, respectively, $\mathbf{t}_{\partial}$ the boundary traction, $\mathbf{S}$ the stress tensor, and $\mathrm{T}^{\mathrm{T}}$ and $\stackrel{\circ}{\mathrm{T}}$ the inner and outer skew-symmetric remodeling couples, respectively. Finally, centered dots and double dots denote the inner products in $\mathbf{V}$ and Lin (the space of 2 nd order tensors), respectively, and $\nabla$ is the gradient operator. Before going further, a couple of remarks are in order. First, the generalized fores entering Eq. (1) belong to two different classes. On the one hand, $\mathbf{b}_{i}, \mathrm{~S}$, and $\mathrm{T}^{\mathrm{T}}$ are inner actions and shall therefore be related to the kinematic fields through thermodynamically consistent constitutive relations. On the other hand, no thermodynamic restrictions apply on the external actions $\mathbf{b}, \mathbf{t}_{\partial}$, and $\stackrel{\circ}{\mathbf{T}}$. In particular, $\stackrel{\circ}{\top}_{\text {encodes the biochemical stimuli directly triggering }}$ remodeling ${ }^{1}$. Second, it can be noticed that this theory is of order 1 in $\mathbf{u}$ and of order 0 in R. Therefore, since no power is expended on the rotation gradients, the rotation of the material axes at one point is insensitive to the rotation at the neighboring points.

The principle of material frame-indifference leads to $\mathbf{b}_{i}=0$ and to the symmetry of the stress tensor $\mathbf{S}$. Hence, the balance laws governing material motion derive from a generalized statement of the principle of virtual power, i.e. from the requirement that the total virtual power-the sum of the powers of internal and external forces-shall be null for any admissible virtual velocity:

$$
\forall(\hat{\mathbf{v}}, \hat{\mathrm{W}}), P_{i}(\hat{\mathbf{v}}, \hat{\mathrm{W}})+P_{e}(\hat{\mathbf{v}}, \hat{\mathrm{W}})=0 .
$$

Replacing the expressions of $P_{i}$ and $P_{e}$ given in Eq. (1) and using the divergence theorem, this reads:

$$
\int_{\mathcal{B}}((\operatorname{div} \mathrm{S}+\mathbf{b}) \cdot \hat{\mathbf{v}}+(\dot{\mathrm{T}}+\stackrel{\circ}{\mathrm{T}}): \hat{\mathrm{W}})+\int_{\partial \mathcal{B}}\left(\mathbf{t}_{\partial}-\mathrm{S} \mathbf{n}_{\partial}\right) \cdot \hat{\mathbf{v}}=0 \quad \forall(\hat{\mathbf{v}}, \hat{\mathrm{W}}),
$$

where div is the divergence operator and $\mathbf{n}_{\partial}$ is the outer unit normal to $\partial \mathcal{B}$.

By standard localization arguments, the integral expression of the principle of virtual power in Eq. (3) leads to the local balance equations:

$$
\begin{aligned}
\operatorname{div} \mathbf{S}+\mathbf{b}=0 \quad \text { in } \mathcal{B}, \quad & \mathbf{S} \mathbf{n}_{\partial}=\mathbf{t}_{\partial} \quad \text { on } \partial \mathcal{B}, \\
\dot{\mathbf{T}}+\stackrel{\circ}{\mathbf{T}}=0 & \text { in } \mathcal{B} .
\end{aligned}
$$

\footnotetext{
${ }^{1}$ As it will be shown later, mechanical forces also do trigger remodeling.
} 


\subsection{Constitutive theory}

Our constitutive theory rests on the specification of the strain energy of the material and on the enforcement of a generalized dissipation principle.

The strain energy $\Psi^{\mathrm{E}}$ is assumed to admit a bulk density $\psi^{\mathrm{E}}$ such that $\Psi^{\mathrm{E}}=\int_{\mathcal{B}} \psi^{\mathrm{E}}$. In turn, the strain energy density $\psi^{\mathrm{E}}$ is postulated as a quadratic form of the small strain tensor $\mathrm{E}$ :

$$
\psi^{\mathrm{E}}=\frac{1}{2} \mathbb{C}(\mathrm{E}): \mathrm{E},
$$

where $\mathbb{C}$ is the 4 th-order elastic tensor. The latter is assumed to be able to evolve in time, which corresponds to material remodeling. Since we focus on rotary remodeling, the actual elastic tensor $\mathbb{C}$ can be obtained through the action of a rotation tensor $\mathrm{R}$ (the kinematic variable describing material remodeling) on a prototype elastic tensor $\mathbb{C}_{0}$, namely:

$$
\forall \mathrm{E}, \mathbb{C}(\mathrm{E})=\mathrm{R} \mathbb{C}_{0}\left(\mathrm{R}^{\top} \mathrm{ER}\right) \mathrm{R}^{\top}
$$

Therefore, the strain energy density $\psi^{\mathrm{E}}$ depends on both the strain $\mathrm{E}$-explicitlyand on the micro-orientation $\mathrm{R}$-implicitly, via $\mathbb{C}$.

In order to simplify the notation, one may use the conjugation product, defined as[59]:

$$
\forall\{A, B, M\} \in \operatorname{Lin},(A \otimes B) M=A M B^{\top} .
$$

Hence, noting that $(A \otimes B)^{\top}=A^{\top} \otimes B^{\top}$ and letting $\mathbb{R}:=R \otimes R$, one can easily recover the following results:

Lemma 2.1.

$$
\begin{aligned}
& \mathrm{R}^{\top} \mathrm{ER}=\mathbb{R}^{\top}(\mathrm{E}), \\
& \mathbb{C}=\mathbb{R} \mathbb{C}_{0} \mathbb{R}^{\top} .
\end{aligned}
$$

Proof. The proof is straightforward.

\subsection{Deriving the local remodeling law from thermody- namics}

Thermodynamic restrictions on the constitutive mappings of the inner actions

$\mathrm{S}$ and $\mathrm{i}$ are obtained by enforcing a generalized statement of the dissipation principle. The latter is a direct consequence of the first and second principles of thermodynamics and binds the power of internal forces $P_{i}$ to the strain energy rate by requiring the intrinsic dissipation $D_{\text {int }}$ to be non-negative along any realized velocity $(\mathbf{v}, \mathrm{W})$ :

$$
D_{i n t}:=-P_{i}(\mathbf{v}, \mathrm{W})-\dot{\Psi}^{\mathrm{E}} \geq 0 .
$$

It can be shown that the rate of strain energy density change can be written as:

$$
\dot{\psi}^{\mathrm{E}}=\frac{1}{2}(\mathbb{C}(\mathrm{E}): \mathrm{E})=\mathbb{C}(\mathrm{E}):\left(\dot{\mathrm{E}}-\left[\dot{\mathrm{R}} \mathrm{R}^{\top}, \mathrm{E}\right]\right)=\mathbb{C}(\mathrm{E}): \dot{\mathrm{E}}-[\mathbb{C}(\mathrm{E}), \mathrm{E}]: \dot{\mathrm{R}} \mathrm{R}^{\top},
$$


where the brackets denote the commutator operator: $\forall(A B) \in \operatorname{Lin},[A, B]=$ $A B-B A=2 \operatorname{skw}(A B)$. Thus, in view of the definition of the inner power in Eq. (1), the local form of Eq. (10) expands to:

$$
(S-\mathbb{C}(E)): \dot{\mathrm{E}}+(-\dot{\mathrm{T}}+[\mathbb{C}(\mathrm{E}), \mathrm{E}]):\left(\dot{\mathrm{R}} \mathrm{R}^{\top}\right) \geq 0 .
$$

No dissipation is assumed to be related to the gross (elastic) deformation, leading to $\mathbf{S}=\partial \psi^{\mathrm{E}} / \partial \mathrm{E}=\mathbb{C}(\mathrm{E})$. Note that $\mathbf{S}$ depends on both $\mathbf{E}$-explicitly-and $\mathbf{R}-$ implicitly, via $\mathbb{C}$, see Eq. (9). Therefore, the local form of the reduced dissipation inequality reads:

$$
\left([\mathrm{S}, \mathrm{E}]-\dot{\mathrm{i}}^{\mathrm{T}}\right):\left(\dot{\mathrm{R}} \mathrm{R}^{\top}\right)=\stackrel{+}{\mathrm{T}}:\left(\dot{\mathrm{R}} \mathrm{R}^{\top}\right) \geq 0
$$

where $\stackrel{+}{\mathrm{T}}:=[\mathrm{S}, \mathrm{E}]-\stackrel{\mathrm{i}}{\mathrm{T}}$ denotes the dissipation couple related to remodeling. For the sake of simplicity, let us assume ${ }^{+}$to be proportional to the remodeling velocity:

$$
\stackrel{+}{\mathrm{T}}=\mathbb{D}\left(\dot{\mathrm{R}} \mathrm{R}^{\top}\right)
$$

where $\mathbb{D}$ represents the 4 th-order dissipation tensor which can be interpreted as a resistance to remodeling. Note that $\mathbb{D}$ belongs to $S k w \otimes S k w$-the vector space of endomorphisms of Skw-and therefore admits the following representation:

$$
\mathbb{D}=\sum_{i j} d_{i j} \mathrm{~W}_{i} \otimes \mathrm{W}_{j}
$$

where $\{i, j\} \in\{\mathrm{x}, \mathrm{y}, \mathrm{z}\}, d_{i j}$ are scalar coefficients and the tensors $\mathrm{W}_{i}$ constitute a basis of Skw, namely: $W_{x}:=\mathbf{e}_{3} \otimes \mathbf{e}_{2}-\mathbf{e}_{2} \otimes \mathbf{e}_{3}, W_{y}:=\mathbf{e}_{1} \otimes \mathbf{e}_{3}-\mathbf{e}_{3} \otimes \mathbf{e}_{1}$, and $\mathrm{W}_{\mathbf{z}}:=\mathbf{e}_{2} \otimes \mathbf{e}_{1}-\mathbf{e}_{1} \otimes \mathbf{e}_{2}$. Moreover, in order for the reduced dissipation inequality in Eq. (13) to be satisfied, $\mathbb{D}$ must be non-negative on any skewsymmetric tensor: $\mathbb{D}(\mathrm{W}): \mathrm{W} \geq 0 \forall \mathrm{W} \in \mathrm{Skw}$, which implies the coefficients $d_{i i}$ to be non-negative and the other coefficients to be null. Thus, the reduced form of $\mathbb{D}$ reads:

$$
\mathbb{D}=\sum_{i} d_{i i} \mathrm{~W}_{i} \otimes \mathrm{W}_{i}
$$

Thus, a thermodynamically-consistent remodeling evolution law is readily obtained, reading:

$$
\mathbb{D}\left(\dot{R} R^{\top}\right)=[S, E]-\stackrel{\circ}{.}
$$

\subsection{Passive remodeling and remodeling equilibrium}

A simple but significant case is obtained by considering the outer remodeling couple $\stackrel{\circ}{T}$ to be null. In this situation, remodeling is only driven by the stress and strain and is therefore called passive remodeling. For the sake of simplicity, we further assume that the dissipation tensor is constant: $\mathbb{D}=\mathbb{D}_{0}$. Hence, at 
each material point, the rotation of the material axes is coupled to the stress and strain by the following equation:

$$
\mathbb{D}_{0}\left(\dot{\mathrm{R}} \mathrm{R}^{\mathrm{T}}\right)=[\mathrm{S}, \mathrm{E}] .
$$

It follows that, provided the material properties $\mathbb{C}_{0}$ and $\mathbb{D}_{0}$, the rotation rate $\dot{\mathrm{R}}$ only depends on the strain $\mathrm{E}$ and the micro-orientation $\mathrm{R}$.

Remodeling equilibrium is achieved when material properties no longer evolve, corresponding to a stationary state of the rotation, i.e. $\dot{\mathrm{R}}=0$. In the case of passive remodeling, it is worth noting that this model predicts the principal axes of the strain and stress tensors to be locally collinear at the remodeling equilibrium[60]. This condition is similar to that inferred by Cowin for trabecular bone[17]. In line with the rationale of the Wolff's law[8], Cowin assumed that trabecular bone remodels itself by stiffening in load bearing directions and concluded that this assumption implies that the principal axes of the stress, strain and fabric tensors all coincide at remodeling equilibrium. Here, we recover the alignment of the principal directions of stress and strain without any $a d$-hoc assumption. However, our model does not necessarily require the material principal axes to align.

\subsection{Comparison with other modeling approaches}

As previously mentioned, a couple of studies aim at describing bone rotary remodeling while acknowledging the role of dissipation in this process[15, 45]. These approaches describe the evolution of the fabric tensor orientation and bone density under certain criteria defining the dead zone, where by definition there is no (or little) remodeling. Jacobs and collaborators[15] use an efficiency measure defined as the difference between the power induced by external actions and the change in total internal energy. In other words, their remodeling law relies on Kuhn-Tucker conditions deriving from the minimization of the dissipation. This global optimization leads to an evolution law for the elastic moduli and bone density. While Jacobs and coworkers use the fourth-order stiffness tensor $\mathbb{C}$ and the density $\rho$ as the variables describing bone remodeling, Doblaré and García[45] account instead for the fabric tensor $\widehat{\mathrm{H}}$ (and therefore, tissue orientation) and the density $\rho$, arguing that the evolutions of $\mathbb{C}$ and $\rho$ are directly coupled in Jacobs et al's model. By describing remodeling through damage theory, they introduce a remodeling tensor $\mathrm{H}$ entering the definition of the remodeling stimulus. Hence, the remodeling law is derived from multi-surface plasticity theory, using criteria on the stimulus for resorption and formation as the boundaries of the dead zone.

The model originally developed by Di Carlo and collaborators[1] that we investigate here lies within the framework of generalized continuum mechanics. This approach relies on balance laws (Eq. (4)) obtained for all the variables through a generalized statement of the virtual power principle (Eq. (3)). A generalized dissipation principle is enforced (Eq. (10)) to obtain strict conditions on the admissible constitutive laws. This approach is essentially different from 
that of Doblaré and García, as they consider the remodeling tensor as an internal variable, whose evolution is represented by the damage tensor. The latter is associated to bone resorption and apposition, leading to the possibility of both increase and decrease of the damage. This feature, which is not considered in classical damage mechanics, opens the possibility for the mechanical dissipation to take negative values. Meanwhile, generalized continuum mechanics ensures the positivity of the dissipation as an intrinsic feature. In other words, in the present work, since our model derives from energetic considerations, the evolution of the fabric abides the laws of thermodynamics.

Moreover, differently from Jacobs et al' approach, we do not require the dissipation to be minimized but simply relate it to remodeling (by assuming a linear relationship with the remodeling rate, see Eq. (14)). Hence, no optimization is enforced: the minimization is here merely a result of the remodeling process since the dissipation-which takes positive values during the remodeling process-decreases to zero as the material remodels towards equilibrium.

Eventually, unlike the aforementioned models, we recall that our model does not require the microstructure to be aligned with the principal stress an strain directions at the remodeling equilibrium. This feature will be illustrated by means of numerical simulations in the Sec. 4 .

\section{Energetic characterization of the rotary re- modeling equilibrium}

\subsection{Extremal properties of the rotary remodeling equilib- rium}

In the present section, we show that, when considering a rotation of the material axes around a fixed axis, equilibrium states are closely bound to the variations of the strain and complementary energy with respect to the rotation angle.

Let us consider a fixed orthonormal frame $\mathbf{e}_{i}$, with $i=1 \ldots 3$. We assume for the sake of simplicity and without loss of generality - that the fixed rotation axis is $\mathbf{e}_{3}$ (see Appendix A in Sec. 6 for the general case of any fixed axis of the $3 \mathrm{D}$ space). In this situation, the rotation tensor $\mathrm{R}$ is parametrised by one scalar-the angle $\alpha$ describing the orientation of the material axes in the plane $\left(\mathbf{e}_{1}, \mathbf{e}_{2}\right)$. Thus, the strain energy density is a function of two variables: the small strain tensor $\mathrm{E}$ and the angle $\alpha$, reading: $\psi^{\mathrm{E}}:(\mathrm{E}, \alpha) \mapsto \psi^{\mathrm{E}}(\mathrm{E}, \alpha)$. Similarly, the complementary energy density is a function of the stress tensor $\mathrm{S}$ and of the angle $\alpha$, reading: $\psi^{\mathrm{S}}:(\mathrm{S}, \alpha) \mapsto \psi^{\mathrm{S}}(\mathrm{S}, \alpha)$.

A sound criterion to detect the remodeling equilibrium can be expressed in terms of the partial derivative of the strain and complementary energy densities with respect to $\alpha$. To this aim, let us first establish the following result which provides an explicit expression of the above derivatives:

Proposition 3.1. The derivatives of the potential and complementary energy 
densities with respect to the rotation angle read:

$$
-\frac{\partial \psi^{\mathrm{E}}}{\partial \alpha}=\frac{\partial \psi^{\mathrm{S}}}{\partial \alpha}=2(\mathrm{SE}): \mathrm{W}_{\mathbf{z}}
$$

Proof. Let $\mathrm{R}$ be a rotation around the axis $\mathbf{e}_{3}$ and $\mathbb{R}=\mathrm{R} \otimes \mathrm{R}$. We refer the reader to lemmas 7.1, 7.2 and 7.3 in Appendix B (Section 7) for keys to the demonstration.

In view of Eq. (5), the partial derivative of the strain energy density with respect to the rotation angle $\alpha$ reads:

$$
\begin{aligned}
\frac{\partial \psi^{\mathrm{E}}}{\partial \alpha}=\frac{1}{2} \frac{\partial \mathbb{C}}{\partial \alpha}(\mathrm{E}): \mathrm{E} & =\frac{1}{2} \frac{\partial}{\partial \alpha}\left(\mathbb{R} \mathbb{C}_{0} \mathbb{R}^{\top}\right)(\mathrm{E}): \mathrm{E} \\
& =\frac{1}{2}\left(\frac{\partial \mathbb{R}}{\partial \alpha} \mathbb{C}_{0} \mathbb{R}^{\top}+\mathbb{R} \mathbb{C}_{0} \frac{\partial \mathbb{R}^{\top}}{\partial \alpha}\right)(\mathrm{E}): \mathrm{E} .
\end{aligned}
$$

We can now calculate separately the two terms of the sum appearing in this expression. The first term can be expressed as follows:

$$
\begin{aligned}
\frac{1}{2}\left(\frac{\partial \mathbb{R}}{\partial \alpha} \mathbb{C}_{0} \mathbb{R}^{\top}\right)(\mathrm{E}): \mathrm{E} & =\frac{1}{2} \frac{\partial \mathbb{R}}{\partial \alpha}\left(\mathbb{C}_{0} \mathbb{R}^{\top}(\mathrm{E})\right): \mathrm{E} \\
& =\operatorname{sym}\left(\mathrm{W}_{\mathrm{z}} \mathbb{R} \mathbb{C}_{0} \mathbb{R}^{\top}(\mathrm{E})\right): \mathrm{E} \\
& =\mathrm{W}_{\mathrm{z}} \mathrm{S}: \mathrm{E} \\
& =\mathrm{SE}: \mathrm{W}_{\mathrm{z}}^{\top} \\
& =-\mathrm{SE}: \mathrm{W}_{\mathrm{z}},
\end{aligned}
$$

where, in the second equality, we made use of Lemma 7.2. The second term of the sum in Eq. (21) can be expressed as follows:

$$
\begin{aligned}
\frac{1}{2}\left(\mathbb{R} \mathbb{C}_{0} \frac{\partial \mathbb{R}}{\partial \alpha}\right)(\mathrm{E}): \mathrm{E} & =\frac{1}{2} \mathbb{R} \mathbb{C}_{0}\left(\frac{\partial \mathbb{R}}{\partial \alpha}(\mathrm{E})\right): \mathrm{E} \\
& =\mathbb{R} \mathbb{C}_{0}\left(\mathrm{~W}_{\mathrm{z}}^{\top} \mathbb{R}^{\top}(\mathrm{E})\right): \mathrm{E} \\
& =\mathbb{R} \mathbb{C}_{0} \mathbb{R}^{\top}\left(\mathrm{W}_{\mathrm{z}}^{\top} \mathrm{E}\right): \mathrm{E} \\
& =\mathrm{W}_{\mathrm{z}}^{\top} \mathrm{E}: \mathrm{S} \\
& =\mathrm{ES}: \mathrm{W}_{\mathrm{z}}
\end{aligned}
$$

where, in the second and third equality, we made use of Lemmas 7.3 and 7.1, respectively.

Finally, combining the results of Eqs. (22-23) and recalling that $\mathrm{W}_{\mathbf{z}}$ is skewsymmetric, we obtain:

$$
\frac{\partial \psi^{\mathrm{E}}}{\partial \alpha}=-(\mathrm{SE}-\mathrm{ES}): \mathrm{W}_{\mathbf{z}}=-2 \mathrm{skw}(\mathrm{SE}): \mathbf{W}_{\mathbf{z}}=-2(\mathrm{SE}): \mathbf{W}_{\mathbf{z}}
$$

Note that, in this expression, the stress $\mathrm{S}$ is meant to be calculated from the strain $E$ as $S=\mathbb{C}(E)=\mathbb{R} \mathbb{C}_{0} \mathbb{R}^{\top}(E)$. 
An equivalent result can be obtained in terms of the complementary energy $\Psi^{\mathrm{S}}$. Let us assume $\Psi^{\mathrm{S}}=\int_{\mathcal{B}} \psi^{\mathrm{S}}$ and postulate the bulk density $\psi^{\mathrm{S}}$ as a quadratic form of the stress tensor $S$ :

$$
\psi^{\mathrm{S}}=\frac{1}{2} \mathbb{C}^{-1}(\mathrm{~S}): \mathrm{S},
$$

where $\mathbb{C}^{-1}$ is the 4 th-order compliance tensor. Hence, using a similar rationale to the one leading to Eq. (24), we eventually obtain:

$$
\frac{\partial \psi^{\mathrm{S}}}{\partial \alpha}=2(\mathrm{SE}): \mathbf{W}_{\mathbf{z}}
$$

where the strain $E$ is meant to be calculated from the stress $S$ as $E=\mathbb{C}^{-1}(S)=$ $\mathbb{R} \mathbb{C}_{0}^{-1} \mathbb{R}^{\top}(\mathrm{S})$.

Finally, Eq. (19) readily follows from Eq. (24) and Eq. (26).

Corollary 3.1. Characterization of the rotary remodeling equilibrium

The rotary remodeling equilibrium is characterized by the following extremal conditions:

$$
\frac{\partial \psi^{\mathrm{E}}}{\partial \alpha}=0 \quad \Leftrightarrow \quad \frac{\partial \psi^{\mathrm{S}}}{\partial \alpha}=\left.0 \quad \Leftrightarrow \quad[\mathrm{S}, \mathrm{E}]\right|_{\mathcal{P}_{\mathbf{e}^{\frac{1}{3}}}}=0,
$$

where, in the last equality, the commutator $[\mathrm{S}, \mathrm{E}]$ is restricted to $\mathcal{P}_{\mathbf{e}_{\frac{1}{3}}}$ - the plane orthogonal to the rotation axis $\mathbf{e}_{3}$.

Proof. The criterion for remodeling equilibrium given in Eq. (27) readily follows from Eq. (19) by noting that $2(\mathrm{SE}): \mathrm{W}_{\mathrm{z}}=2 \operatorname{skw}(\mathrm{SE}): \mathrm{W}_{\mathrm{z}}=[\mathrm{S}, \mathrm{E}]: \mathrm{W}_{\mathrm{z}}$ and that, in this inner product, only the restriction of $[S, E]$ to the plane $\mathcal{P}_{\mathbf{e}_{\frac{1}{3}}^{+}}$ matters.

It is worth noting that a classical mechanical result is recovered in the above characterization, namely that (remodeling) equilibrium states correspond to (partial) extrema of the strain and complementary energy.

\subsection{Stability of equilibrium states}

The stability of the equilibrium states identified in Sec. 3.1 can be assessed by observing the evolution of the material axes close to these states. As in Sec. 3.1, let us assume that the material axes can rotate around the direction $\mathbf{e}_{3}$ and call $\alpha$ their current orientation. An orientation $\alpha^{\mathrm{SE}}$ will be said to correspond to a stable equilibrium (SE) configuration if it is a local attractor-i.e., if the material axes will tend to align as per $\alpha^{\mathrm{SE}}$ when they are oriented as per $\alpha$ close to $\alpha^{\mathrm{SE}}$.

The goal of this section is to characterize these stable equilibrium states using an energetic argument. Let us first consider the following proposition: 
Lemma 3.1. For a given strain $\mathrm{E}$, the derivative of the potential energy is proportional to the rotation rate:

$$
\frac{\partial \psi^{\mathrm{E}}}{\partial \alpha}=-2 d_{0} \dot{\alpha}
$$

Proof. Recall from Proposition 3.1 that:

$$
\frac{\partial \psi^{\mathrm{E}}}{\partial \alpha}=-2(\mathrm{SE}): \mathrm{W}_{\mathbf{z}}=-[\mathrm{S}, \mathrm{E}]: \mathrm{W}_{\mathbf{z}}
$$

The remodeling law given in Eq. (18) provides:

$$
\frac{\partial \psi^{\mathrm{E}}}{\partial \alpha}=-\mathbb{D}_{0}\left(\dot{\mathrm{R}} \mathrm{R}^{\top}\right): \mathbf{W}_{\mathbf{z}}
$$

Noting that $\dot{R} R^{\top}=\dot{\alpha} W_{z}$, only one coefficient of $\mathbb{D}_{0}$ matters in the above scalar product, namely $d_{z z}$. Renaming this coefficient $d_{0}$, Eq. (28) is readily obtained.

For a given strain $\mathrm{E}$, the values taken by the strain energy and its derivatives depend on the orientation of the microstructure, i.e. the angle $\alpha$. This allows us to express the stability of an equilibrium state in terms of convexity of the strain energy with respect to $\alpha$. Let us first introduce the following proposition:

Proposition 3.2. An orientation $\alpha^{\mathrm{SE}}$ corresponds to a stable equilibrium configuration if and only if

$$
\frac{\partial^{2} \psi^{\mathrm{E}}}{\partial \alpha^{2}}\left(\alpha^{\mathrm{SE}}\right)>0
$$

Proof. For a given strain E, let us introduce the function $\rho=\alpha \mapsto \rho(\alpha)=$ $-[\mathrm{S}(\mathrm{E}, \alpha), \mathrm{E}]: \mathrm{W}_{\mathbf{z}}$. Thus, Eq. (19) can be rewritten as:

$$
\frac{\partial \psi^{\mathrm{E}}}{\partial \alpha}=\rho
$$

It is worth noting that the rotary equilibrium configurations correspond to the roots of $\rho$ since this latter is noting but the rotation rate, but a negative coefficient-see Eq. (28). In view of Lemma 3.1, the following identity holds at any time $t$ :

$$
\rho(\alpha(t))=-2 d_{0} \dot{\alpha}(t) .
$$

Let us assume that $\alpha^{\mathrm{SE}}$ corresponds to a stable equilibrium configuration for the material. Thus, if the orientation $\alpha$ of the material axes is slightly shifted from $\alpha^{\mathrm{SE}}$, the material axes will rotate towards $\alpha^{\mathrm{SE}}$. In particular, if $\alpha<\alpha^{\mathrm{SE}}$, $\alpha$ will increase toward $\alpha^{\mathrm{SE}}$ and then $\dot{\alpha}>0 \equiv \rho(\alpha)<0$. Conversely, if $\alpha>\alpha^{\mathrm{SE}}$, 
$\alpha$ will decrease toward $\alpha^{\mathrm{SE}}$ and then $\dot{\alpha}<0 \equiv \rho(\alpha)>0$. Provided that the function $\rho$ is regular enough, this implies that:

$$
\frac{\partial \rho}{\partial \alpha}\left(\alpha^{\mathrm{SE}}\right)>0
$$

and therefore Eq. (30) holds true.

On the other way around, Eq. (30) implies, on the one hand, that $\rho(\alpha)<0$ and thus $\dot{\alpha}>0$ if $\alpha<\alpha^{\mathrm{SE}}$; and, on the other hand, that $\rho(\alpha)>0$ and thus $\dot{\alpha}<0$ if $\alpha>\alpha^{\mathrm{SE}}$. (In both cases, $\alpha$ is assumed to lie in a neighborhood of $\alpha^{\mathrm{SE}}$.) In turn, this implies the stability of $\alpha^{\mathrm{SE}}$.

Proposition 3.2 and Eq. (19) allow us to deduce the following result:

Proposition 3.3. An orientation $\alpha$ corresponds to a stable equilibrium configuration if and only if

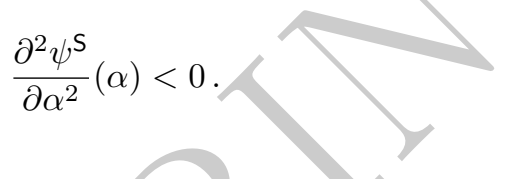

Thus, in view of Propositions 3.2 and 3.3, it is possible to state the following key characterization:

Corollary 3.2. Characterization of stable rotary remodeling states

$$
\begin{aligned}
& \alpha^{\mathrm{SE}} \text { stable equilibrium } \Leftrightarrow \text { local minimum of } \alpha \mapsto \psi^{\mathrm{E}} \Leftrightarrow \text { local maximum of } \\
& \alpha \mapsto \psi^{\mathrm{S}}
\end{aligned}
$$

As a conclusion, we retrieve another classical result from mechanics, which is that stable equilibrium states correspond to minima of the potential energy and to maxima of the complementary energy.

\section{Numerical assessment of the influence of load- ing conditions and material properties on the remodeling}

Numerical simulations were performed to study the response of the model and the remodeling equilibrium states with respect to different loading conditions and for different elastic moduli. Without lack of generality, we assume hereinafter that the material axes rotate around the axis $\mathbf{e}_{3}$. Therefore, the problem will be studied in the $2 \mathrm{D}$ plane $\left(\mathbf{e}_{1}, \mathbf{e}_{2}\right)$.

As in DiCarlo et al[1], the material is assumed to be transversely isotropic, the axis $\mathbf{e}_{1}$ being orthogonal to the isotropy plane. The relevant elastic moduli, adapted from [61], are summarized in Table 1 with the Kelvin notation. Three types of materials are considered in this study, characterized by high (A), mild (B), and low (C) values of the shear modulus $C_{66}$. 
Table 1: Two-dimensional material elastic properties (compressed Kelvin notation).

\begin{tabular}{cccccccc}
\hline Material type & $C_{11}$ & $C_{22}$ & $C_{12}$ & $C_{16}$ & $C_{26}$ & $C_{66}$ & $d_{0}$ \\
\hline $\mathrm{A}$ & & & & & & $20 \mathrm{GPa}$ & \\
$\mathrm{B}$ & $30 \mathrm{GPa}$ & $20.85 \mathrm{GPa}$ & $11.49 \mathrm{GPa}$ & $0 \mathrm{GPa}$ & $0 \mathrm{GPa}$ & $13.2 \mathrm{GPa}$ & $555 \mathrm{kPa}$. day \\
$\mathrm{C}$ & & & & & & $2 \mathrm{GPa}$ & \\
\hline
\end{tabular}

In the following sections, we first analyze the application of a uniaxial stress in the $\mathbf{e}_{1}$ direction: we depict the variations of the strain energy with respect to the material orientation and show how its evolution can lead to different equilibrium states when changing the initial orientation or material properties. Furthermore, we discuss the dependency on material properties and loading conditions thanks to analytical and numerical considerations.

\subsection{Equilibrium states and energy}

Numerical simulations were performed to investigate the rotary remodeling of the material in uniaxial, i.e. stress/strain conditions. Fig. 2 shows the results obtained by applying either a uniaxial strain (subplots $(a, b, c)) E=E_{11} \mathrm{e}_{1} \otimes \mathrm{e}_{1}$ or stress (subplots $(\mathrm{d}, \mathrm{e}, \mathrm{f})) \mathrm{S}=S_{11} \mathrm{e}_{1} \otimes \mathrm{e}_{1}$ and for material types A (high shear modulus, subplots (a,d)), B (mild shear modulus, subplots (b,e)) and C (low shear modulus, subplots $(\mathrm{c}, \mathrm{f})$ ), see Table 1 . The function $\rho$ (solid blue lines), the strain energy (brown dotted lines) and the complementary energy (dark green dotted lines) are plotted versus the angle $\alpha$ describing the orientation of the material axes in the plane $\left(\mathbf{e}_{1}, \mathbf{e}_{2}\right)$. As expected, the roots of the function $\rho$ $-i . e .$, the remodeling equilibrium states of the material-correspond to extrema of the potential $(\mathrm{a}, \mathrm{b}, \mathrm{c})$ and complementary $(\mathrm{d}, \mathrm{e}, \mathrm{f})$ energy. Stable roots (green knots) are attained where the potential energy reaches a local minimum (a,b,c) and the complementary energy reaches a local maximum (d,e,f). Conversely, unstable roots (red knots) correspond to local maxima of the potential energy $(a, b, c)$ and local minima of the complementary energy (d,e,f).

Numerical simulations were also performed to investigate the evolution of the strain and complementary energy during remodeling. The effects of the initial orientation $\left(\alpha_{0}\right)$ of the material axes were investigated in uniaxial strain conditions. Whatever the initial orientation of the material axes, the system underwent rotary remodeling until achieving a stable equilibrium configuration. In agreement with the theoretical results, these stable remodeling equilibrium states were found to correspond to minima of the strain energy. Results of these simulations are shown in Fig. 3 where are plotted the time courses of the strain energy for material types A (subplot (a)) and C (subplot (b)) and for different initial orientations of the material axes (uniaxial strain).

For the material type A, characterized by a high shear modulus, two stable equilibrium states are observed: one corresponds to an alignment of the material axes with the loading direction $\left(\alpha^{\mathrm{SE}}=0\right)$, and the other corresponds to a rota- 
tion towards the normal to that direction $\left(\alpha^{\mathrm{SE}}=\frac{\pi}{2}\right)$. The unstable equilibrium states observed in Fig. 2(a) do not appear in this plot. For the material type $\mathrm{C}$, characterized by a low shear modulus, three equilibrium states are found in Fig. 3, corresponding to stable and unstable states observed in Fig. 2(c). One can note that $\alpha=0$ and $\alpha=\frac{\pi}{2}$ are not stable and are attained only by assigning either of these values as initial condition for $\alpha_{0}$. For any other initial orientation of the material axes, the strain energy would tend to another-stable-equilibrium state.

\subsection{Influence of material properties and loading condi- tions}

This section is devoted to present and discuss the effects of the material properties and of different loading conditions on the remodeling equilibrium states. As a direct application of Corollaries 3.1 and 3.2, the existence and the stability of equilibrium configurations is assessed searching for the roots of the function $\rho$ (Eq. (31)) and studying the sign of its derivative around the roots.

\subsubsection{On the alignment of the microstructure with principal stresses}

Let us assume our medium to subjected to a uniaxial stress $\mathrm{S}=S_{11} \mathrm{e}_{1} \otimes \mathrm{e}_{1}$ and the stiffest direction to be initially oriented along the stress axis $\mathbf{e}_{1}\left(\alpha_{0}=0\right)$. Hence, we investigated the relationships between the elastic coefficients of the material and the stability of this configuration.

In this context, the function $\rho$ reads:

$$
\rho(\alpha)=-\frac{\partial \psi^{\mathrm{S}}}{\partial \alpha}(\alpha)=\frac{S_{11}^{2}}{\operatorname{det} \mathbb{C}_{0}}\left(f_{1}\left(\mathbb{C}_{0}\right) \sin 2 \alpha+\frac{f_{2}\left(\mathbb{C}_{0}\right)}{2} \sin 4 \alpha\right),
$$

where $f_{1}$ and $f_{2}$ are functions of $\mathbb{C}_{0}$ and $\operatorname{det} \mathbb{C}_{0}$ is the determinant of the stiffness tensor (explicit expressions are provided in the Appendix $\mathrm{C}$ in Sec. 8). Hence, since $\rho(0)=0$, we find-in line with Cowin[17]-that the alignment of the material axes with the stress principal direction correspond to a remodeling equilibrium state. However, this configuration is not necessarily stable and the material does not necessarily tend to realign with the stress direction after a perturbation. In order for $\alpha=0$ to be a stable equilibrium state, the derivative of $\rho$ has to be positive. In the present scope, this condition reads:

$$
\frac{\partial \rho}{\partial \alpha}(0)=\frac{S_{11}^{2}}{\operatorname{det} \mathbb{C}_{0}} f_{3}\left(\mathbb{C}_{0}\right)>0,
$$

where $f_{3}$ is a function of $\mathbb{C}_{0}$ (its explicit expression is provided in the Appendix $\mathrm{C}$ in Sec. 8).

Fig. 4 shows the evolution of the function $f_{3}$ by varying one elastic coefficient at once and fixing the other elastic moduli to the values of material A in Table 1. The orientation $\alpha=0$, aligned with principle stresses, is a stable equilibrium only if the function $f_{3}$ stays positive. This is not the case if the transverse 
modulus $C_{22}$ is too high (transverse direction takes over; dash-dotted line in Fig. 4), or either of moduli $C_{66}$ (shear; solid line) or $C_{12}$ (axial coupling; dashed line) is too low. Hence, we find here a strong dependency of the stability of the configuration $\alpha=0$ on the material properties.

\subsubsection{Numerical identification of equilibrium states}

Expanding the analysis of the previous section, we investigated the effects of the loading conditions and of the shear modulus on the remodeling equilibrium states. Let us assume the material axes to be initially oriented along the axis $\mathbf{e}_{1}$, i.e. $\alpha_{0}=0$. It was shown in the previous section that this is either a stable or an unstable remodeling equilibrium configuration in uniaxial loading conditions. It is interesting to study the response of the material and the new remodeling equilibrium configuration-i.e. the closest stable equilibrium state to 0 -that it would reach due to a change of the loading conditions. For the sake of simplicity, only two types of loading conditions, namely biaxial plane stress and combined axial/shear plane stress in the plane $\left(\mathbf{e}_{1}, \mathbf{e}_{2}\right)$, and the three material types in Table 1 are considered here. Results of this study are shown in Fig. 5, where the effects of the two loading conditions are investigated independently, and in Fig. 6, which refers to a general 2D stress state.

In the case of longitudinal axial loading $\left(S_{11}=1 \mathrm{MPa}, S_{12}=0, S_{22}=0\right)$, both Fig. 5.a and 5.b indicate that $\alpha=0$ is a stable state only for high shear moduli (material type A). For lower shear moduli (material properties B,C), the material remodels by rotating its axes toward an orientation $\alpha^{\mathrm{SE}} \in\left[\frac{\pi}{4}, \frac{\pi}{2}\right]$. These results confirm what has been observed in Fig. 2.

On the one hand, when only shear and longitudinal stress are to account for $\left(S_{22}=0\right.$, Fig. $\left.5(\mathrm{~b})\right)$, the function $\rho$ reads:

$$
\rho(\alpha)=\left(S_{11} \sin 2 \alpha-S_{12} \cos 2 \alpha\right)\left(f_{1}\left(\mathbb{C}_{0}\right) S_{11}+f_{2}\left(\mathbb{C}_{0}\right)\left(S_{11} \cos 2 \alpha+S_{12} \sin 2 \alpha\right)\right)
$$

Hence, the addition of shear necessarily leads to a change in the principal stress directions and therefore shifts the equilibrium state $(\rho(\alpha)=0)$, as suggested by Pedersen[33]. This result can be visualized in Fig. 5 (b): the closest stable equilibrium to $\alpha=0$ is never 0 , except if, as shown in Fig. 6 , the addition of a transverse axial stress compensates this effect. Moreover, as one could predict, there is a central symmetry around $\left\{S_{12}=0, \alpha=0\right\}$ as the equilibrium state is symmetrical with respect to $S_{12}=0: \alpha_{\text {right }}^{\mathrm{SE}}=-\alpha_{\text {left }}^{\mathrm{SE}}$.

On the other hand, for a configuration involving both longitudinal and transverse axial stress but no shear stress $\left(S_{12}=0\right.$, Fig. 5 (a)), the dependency of $\rho$ on the material properties is more complex. In this case, very high values of the ratio $\frac{S_{22}}{S_{11}}$ depict a loading configuration similar to a simple traction or compression along the transverse axis (negligible longitudinal loading and no shear loading). In that configuration, $\alpha=0$ is a stable equilibrium for high and mild shear moduli (material types $\mathrm{A}$ and $\mathrm{B}$ ), and $\alpha^{\mathrm{SE}} \in\left[0, \frac{\pi}{4}[\right.$ for low shear moduli (material type C).

Fig. 6 displays three 3D plots giving the closest stable equilibrium states 
to 0 as a function of both $S_{12}$ and $S_{22}$ for the 3 material types (A, B, C) in Table 1. The lines depicted in Fig. 5 are superimposed on the surfaces using the same line colors and styles. These results underline the complexity of the coupling between the loading components in how they affect remodeling. Moreover, it is apparent that the material response strongly depends on the value of the shear modulus (subplot (a): material A, high shear modulus; (b): material B, mild shear modulus; (c): material C, low shear modulus). Recalling that $\alpha=0$ corresponds to a configuration where the material axes are aligned with the longitudinal stress, it can be noticed that the latter (described by the white areas of the surfaces) is a stable remodeling equilibrium state only in very special conditions depending on both the loading conditions and the material properties.

\subsection{Mixed boundary conditions}

Because in vivo loading conditions are complex, living tissue is subjected to mixed boundary conditions and an inhomogeneous stress/strain state. Hence, the evolution of the system is more complex and the local minimization and maximization principles derived in Section 3 do not apply any more.

In order to describe the adaptation of bone in a complex loading configuration, we coupled the remodeling law (Eq. (17)) to a finite element analysis, using Comsol with Matlab v5.3a. We subjected the 2D structure displayed in Fig. 7, a toy model of a human proximal femur, to loading and boundary conditions approximating in vivo conditions, as per Beaupré et al[62]. The cortical tissue (plain black) was assumed isotropic with a Young's modulus $E_{c}=14 \mathrm{GPa}$ and a Poisson's ratio $\nu_{c}=0.3$. Trabecular bone was assumed to be transversely isotropic and undergoing rotary remodeling, with $C_{11}=2.50 \mathrm{GPa}$, $C_{22}=1.42 \mathrm{GPa}, C_{12}=636 \mathrm{MPa}$ and $C_{66}=1.20 \mathrm{GPa}$. Figure 7 describes the evolution of the micro-orientation in the proximal femur. An arbitrary, yet reasonable initial micro-orientation field was assigned (see Fig. 7(a)). Starting from this configuration, material remodeling changes the microstructural alignment in response to the loading conditions. Despite its simplicity, the model provides a reasonable distribution of the micro-orientation after remodeling.

\section{Conclusions}

In this paper, we described a thermodynamically consistent model for orthotropic bone remodeling and showed its ability to describe the evolution of material axes in 2D. In particular, we demonstrated that the remodeling response of the material is highly dependent on its material properties and on the loading conditions.

In the present study, we demonstrated that the model retrieves classical energy theorems. In particular, stable remodeling equilibrium states have been shown to correspond to local minima of the strain energy (in strain-controlled loading conditions) and maxima of the complementary energy (in stress-controlled boundary conditions). The authors stress the importance of the local aspect of 
the energy theorems demonstrated in Section 3: the energetic theorems demonstrated previously are valid solely in the case of homogeneous and stationary stresses or strains. Indeed, in uniform strain conditions, the stress experienced by the material evolves in time with the reorientation of the material axes but the strain stays stationary. Conversely, in uniform stress conditions, the strain evolves in time but the stress stays stationary. If mixed boundary conditions are applied (as, for example, in boundary value problems where both displacements and stress may be applied on the boundary of the system), the evolution of the system is more complex as the environment surrounding a point is constantly evolving. Furthermore, typically, neither the strain nor the stress are stationary: the evolution of the strain energy is therefore more complex and no energetic theorems have been obtained so far for general boundary conditions.

The model delivered sound results on the influence of material properties and boundary conditions.

First, we have shown that the mathematical condition for remodeling equilibrium described by Cowin[17] - i.e. the alignment of principal stress, principal strain and material directions - is attained only in special cases, namely for sufficiently high values of the shear modulus. Otherwise, only the first part of this statement holds true but the material axes do not necessarily align with the principal stress and strain directions at the remodeling equilibrium. Second, we have highlighted the strong effects of the mechanical environment and of the material properties on remodeling equilibrium. On the one hand, the addition of shear or transverse stress strongly modifies the remodeling equilibrium configuration. On the other hand, the material properties themselves play a role in the achievement of the equilibrium as the alignment of stresses and strains merely ensure to have reached an equilibrium but not whether it is stable or not.

The formulation of a thermodynamically-driven process has the potential to model complex processes, like bone remodeling, which involve mechanical as well as biochemical regulations. Furthermore, the constant evolution of living tissue implies that remodeling equilibrium is a global point of view as homeostasis remains a dynamic state.

\section{Acknowledgments}

This project has received funding from the European Research Council (ERC) under the European Union's Horizon 2020 research and innovation program (grant agreement No 682001, project ERC Consolidator Grant 2015 BoneImplant). 


\section{Appendix A: From a rotation around the Z- axis to a rotation around any fixed axis in the $3 \mathrm{D}$ space}

Corollary 6.1. Generalization of Proposition 19 to ANy FiXed AXis OF THE 3D SPACE.

Proposition 19 can be extended to the case of any fixed axis $\mathbf{u}$ in the $3 D$ space as follows:

$$
\frac{\partial \psi^{\mathrm{E}}}{\partial \alpha}=2\left(\mathrm{E}_{\mathrm{P}} \cdot \mathrm{S}_{\mathrm{P}}\right):\left(\mathrm{W}_{\mathbf{z}}\right)
$$

where $\mathrm{P}$ is the (unique) rotation that transforms $\mathbf{u}$ into $\mathbf{e}_{3}$ and $\mathrm{E}_{\mathrm{P}}=\mathrm{PEP}^{\top}$, $\mathrm{S}_{\mathrm{P}}=\mathrm{PSP}^{\top}$ and $\mathcal{P}_{\mathbf{u}}=\operatorname{Vect}(\mathbf{u} \otimes \mathbf{u})^{\perp}$, leading to the criterion for equilibrium:

$$
\frac{\partial \psi^{\mathrm{E}}}{\partial \alpha}=\left.0 \Leftrightarrow\left[\mathrm{S}_{\mathrm{P}}, \mathrm{E}_{\mathrm{P}}\right]\right|_{\mathcal{P}_{\mathbf{u}}}=0
$$

Proof. We define here the (unique) rotation $\mathrm{P}$ that transforms $\mathbf{u}$ into $\mathbf{e}_{3}$.

This rotation defines a new frame $\mathcal{R}_{X^{\prime} Y^{\prime} Z^{\prime}}$. Subsequently, we can determine the rotation $R_{0}$ around $\mathbf{e}_{3}$ such that $R_{0}=P . R . P^{\top}=\mathbb{P}[R]$ where $\mathbb{P}=P \otimes P$. Hence, we also have $R=\mathbb{P}^{\top} R_{0}$. The strain energy density reads:

$$
\begin{aligned}
\psi & =\frac{1}{2}(\mathrm{~S}: \mathrm{E})=\frac{1}{2}\left(\mathbb{R} \mathbb{C}_{0} \mathbb{R}^{\top}\right)(\mathrm{E}): \mathrm{E} \\
& =\frac{1}{2}\left(\left(\mathrm{P}^{\top} \cdot \mathrm{R}_{0} \cdot \mathrm{P}\right) \cdot\left(\mathbb{C}_{0}\left[\left(\mathrm{P}^{\top} \cdot \mathrm{R}_{0} \cdot \mathrm{P}\right)^{T} \cdot \mathrm{E} \cdot\left(\mathrm{P}^{\top} \cdot \mathrm{R}_{0} \cdot \mathrm{P}\right)\right] \cdot\left(\mathrm{P}^{\top} \cdot \mathrm{R}_{0} \cdot \mathrm{P}\right)^{T}\right): \mathrm{E}\right. \\
& =\frac{1}{2}\left(\mathbb{P}_{0}\left(\mathbb{P}^{\top} \cdot\left(\mathrm{R}_{0}^{\top} \cdot \mathbb{P}^{\top}(\mathrm{E}) \cdot \mathrm{R}_{0}\right)\right)\right):\left(\mathrm{R}_{0}^{\top} \cdot \mathbb{P}(\mathrm{E}) \cdot \mathrm{R}_{0}\right)
\end{aligned}
$$

We now define the fourth-order tensor $\mathbb{C}_{\mathrm{P}}$ accounting for the endomorphism $\widetilde{\mathbb{C}_{\mathrm{P}}}$ acting in the space of second-order tensors in the following manner: $\widetilde{\mathbb{C}_{\mathrm{P}}}=$ $\mathrm{A} \mapsto \mathbb{P} \mathbb{C}_{0}\left(\mathbb{P}^{\top} \mathrm{A}\right)=\mathbb{P} \mathbb{C}_{0} \mathbb{P}^{\top}(A)$. The tensor $\mathbb{C}_{\mathrm{P}}$ naturally inherits from the symmetries of $\mathbb{C}_{0}$, and represents the stiffness matrix in the new frame $\mathcal{R}_{X^{\prime} Y^{\prime} Z^{\prime}}$. In addition, we define the strain tensor in the rotated frame $E_{\mathrm{P}}=\mathbb{P}^{\top} \mathrm{E}$.

Consequently, we can write the strain energy density as follows:

$$
\psi=\frac{1}{2} \mathbb{C}_{\mathrm{P}}\left(\mathbb{R}_{0}^{\top} \mathrm{E}_{\mathrm{P}}\right):\left(\mathbb{R}_{0}^{\top} \mathrm{E}_{\mathrm{P}}\right)
$$

As a result, finding the extrema of the strain energy is strictly equivalent to finding them for a material with elastic properties $\mathbb{C}_{\mathrm{P}}$ submitted to a strain $E_{\mathrm{P}}$.

Finally, for any fixed axis, the energy reaches an extremum (minimum or maximum) if and only if the restriction of product of the newly-defined rotated stress $S_{\mathrm{P}}=\mathbb{R}_{0}^{\top} \mathbb{C}_{\mathrm{P}} \mathbb{R}_{0}^{\top} \mathrm{E}_{\mathrm{P}}$ and strain $\mathrm{E}_{\mathrm{P}}$ principal axes to the plane $\mathcal{P}_{\mathbf{u}}$ is symmetric. 


\section{Appendix B: Key properties of the rotation and elasticity tensors}

We give here several key properties to ease the reading of the demonstrations in Section 3. Each of the following lemmas can be easily proven by simple algebraic calculations.

Lemma 7.1. Properties of the Rotations.

$$
\begin{aligned}
\mathrm{RW}_{\mathrm{z}} & =\mathrm{W}_{\mathrm{z}} \mathrm{R}, \\
\mathbb{R} \mathrm{W}_{\mathrm{z}} & =\mathrm{W}_{\mathrm{z}}, \\
\mathbb{R}\left(\mathrm{W}_{\mathrm{z}} \mathrm{A}\right) & =\mathrm{W}_{\mathrm{z}} \mathbb{R}(\mathrm{A}) \quad \forall \mathrm{A} \in \operatorname{Lin}, \\
\mathbb{R}\left(\mathrm{AW} \mathrm{W}_{\mathrm{z}}\right) & =\mathbb{R}(\mathrm{A}) \mathrm{W}_{\mathrm{z}} \quad \forall \mathrm{A} \in \operatorname{Lin} .
\end{aligned}
$$

Lemma 7.2. Properties of the Derivatives of the Rotation ténsors.

$$
\begin{aligned}
\frac{\partial R}{\partial \alpha} & =R W_{z}=W_{z} R, \\
\frac{\partial R^{\top}}{\partial \alpha} & =R^{\top} W_{z}^{\top}=W_{z}^{\top} R^{\top}, \\
\frac{\partial \mathbb{R}}{\partial \alpha} & =\frac{\partial}{\partial \alpha}(R \otimes R)=\left(W_{z} R\right) \otimes R+R \otimes\left(W_{z} R\right), \\
\frac{\partial \mathbb{R}^{2}}{\partial \alpha} & =\frac{\partial}{\partial \alpha}\left(R^{\top} \otimes R^{\top}\right)=\left(W_{z}^{\top} R^{\top}\right) \otimes R^{\top}+R^{\top} \otimes\left(W_{z}^{\top} R^{\top}\right), \\
\frac{\partial \mathbb{R}}{\partial \alpha}(A) & =2 \operatorname{sym}\left(W_{z} \mathbb{R}(A)\right) \quad \forall A \in \operatorname{Sym}, \\
\frac{\partial \mathbb{R}^{\top}}{\partial \alpha}(A) & =2 \operatorname{sym}\left(W_{z}^{\top} \mathbb{R}^{\top}(A)\right) \quad \forall A \in \operatorname{Sym} .
\end{aligned}
$$

Lemma 7.3. Properties of tHe Elasticity tensor.

$$
\begin{aligned}
\mathbb{C}\left(\frac{\partial \mathbb{R}}{\partial \alpha}(\mathrm{A})\right) & =2 \mathbb{C}\left(\mathrm{W}_{\mathrm{z}} \mathbb{R}(\mathrm{A})\right) \quad \forall \mathrm{A} \in \mathrm{Lin}, \\
\mathbb{C}\left(\frac{\partial \mathbb{R}^{\top}}{\partial \alpha}(\mathrm{A})\right) & =2 \mathbb{C}\left(\mathrm{W}_{\mathrm{z}}^{\top} \mathbb{R}^{\top}(\mathrm{A})\right) \quad \forall \mathrm{A} \in \mathrm{Lin} .
\end{aligned}
$$

\section{Appendix C: Explicit expressions of the func- tion $\rho$ and of its derivative}

Assuming to apply a uniaxial tensile stress $\mathrm{S}=S_{11} \mathrm{e}_{1} \otimes \mathrm{e}_{1}$, the expression of $\rho$ reads (Eq. (35)):

$$
\rho(\alpha)=\frac{S_{11}^{2}}{\operatorname{det} \mathbb{C}_{0}}\left(f_{1}\left(\mathbb{C}_{0}\right) \sin 2 \alpha+\frac{f_{2}\left(\mathbb{C}_{0}\right)}{2} \sin 4 \alpha\right),
$$

where

$$
\begin{aligned}
f_{1}\left(\mathbb{C}_{0}\right) & =C_{44} C_{55} C_{66}\left(C_{13}^{2}-C_{23}^{2}-C_{11} C_{33}+C_{22} C_{33}\right), \\
f_{2}\left(\mathbb{C}_{0}\right) & =C_{44} C_{55}\left(2 \sqrt{2} C_{11} C_{23}^{2}+2 \sqrt{2} C_{12}^{2} C_{33}-2 \sqrt{2} C_{11} C_{22} C_{33}+C_{13}^{2}\left(2 \sqrt{2} C_{22}-C_{66}\right)\right. \\
& -C_{23}^{2} C_{66}+C_{11} C_{33} C_{66}+2 C_{12} C_{33} C_{66}+C_{22} C_{33} C_{66} \\
& \left.-C_{13} C_{23}\left(2 \sqrt{2} C_{12}+C_{66}\right)\right), \\
\operatorname{det} \mathbb{C}_{0} & =C_{44} C_{55} C_{66}\left(C_{13}^{2} C_{22}-2 C_{12} C_{13} C_{23}+C_{12}^{2} C_{33}+C_{11}\left(C_{23}^{2}-C_{22} C_{33}\right)\right) .
\end{aligned}
$$


In the above expression, $C_{i j}$ represent the coefficients of $\mathbb{C}_{0}$ in the Kelvin notation.

The derivative of $\rho$ reads:

$$
\frac{\partial \rho}{\partial \alpha}=\frac{2 S_{11}^{2}}{\operatorname{det} \mathbb{C}_{0}}\left(f_{1}\left(\mathbb{C}_{0}\right) \cos 2 \alpha+f_{2}\left(\mathbb{C}_{0}\right) \cos 4 \alpha\right) .
$$

In particular:

$$
\frac{\partial \rho}{\partial \alpha}(0)=\frac{2 S_{11}^{2}}{\operatorname{det} \mathbb{C}_{0}}\left(f_{1}\left(\mathbb{C}_{0}\right)+f_{2}\left(\mathbb{C}_{0}\right)\right)=\frac{2 S_{11}^{2}}{\operatorname{det} \mathbb{C}_{0}} f_{3}\left(\mathbb{C}_{0}\right)
$$

where

$$
\begin{aligned}
f_{3}\left(\mathbb{C}_{0}\right)= & 2\left(f_{1}\left(\mathbb{C}_{0}\right)+f_{2}\left(\mathbb{C}_{0}\right)\right) \\
= & 2 C_{44} C_{55}\left(\sqrt{2} C_{13}^{2} C_{22}+\sqrt{2} C_{12}^{2} C_{33}+\sqrt{2} C_{11}\left(C_{23}^{2}-C_{22} C_{33}\right)\right. \\
& \left.-C_{23}^{2} C_{66}+C_{12} C_{33} C_{66}+C_{22} C_{33} C_{66}-C_{13} C_{23}\left(2 \sqrt{2} C_{12}+C_{66}\right)\right)
\end{aligned}
$$

\section{References}

[1] DiCarlo A, Naili S, Quiligotti S, Sur le remodelage des tissus osseux anisotropes, Comptes Rendus Mécanique 334(11):651-661, 2006.

[2] Frost HM, Bone "mass" and the "mechanostat": A proposal, The Anatomical Record 219(1):1-9, 1987.

[3] Von Meyer GH, Die Architektur der Spongiosa. (Zehnter Beitrag zur Mechanik des menschlichen Knochgerüstes), Archiv fur Anatomie, Physiologie, und wissenschaftliche Medizin 6:615-628, 1867.

[4] Wolff J, Ueber die innere Architectur der Knochen und ihre Bedeutung für die Frage vom Knochenwachsthum, Virchows Archiv Pathol Anat Physio 50:389-450, 1870 .

[5] Roux W, Der Kampf der Teile des Organismus, Engelmann, Leipzig, 1881.

[6] Culmann K, Die graphische Statik, Meyer \& Zeller (A . Reimann), Zürich, 1866.

[7] Wolff J, Das Gesetz der Transformation der Knochen, Hirschwald, Berlin, Germany, 1892.

[8] Wolff J, The classic: on the inner architecture of bones and its importance for bone growth. 1870, Clinical orthopaedics and related research 468(4):1056-1065, 2010.

[9] Garden, RS, The Structure and Function of the Proximal End of the Femur, Journal of Bone and Joint Surgery 43-B(3): 576-589, 1961. 
[10] Singh M, Nagrath AR, Maini PS, Changes in trabecular pattern of the upper end of the femur as an index of osteoporosis, Journal of Bone and Joint Surgery, 52(3): 457--67, 1970.

[11] Whitehouse WJ, The quantitative morphology of anisotropic trabecular bone, Journal of microscopy 101(Pt 2):153-68, 1974.

[12] Takechi H, Trabecular Architecture of the Knee Joint, Acta Orthopaedica, 48(6): 673-681, 1977.

[13] Tobin WJ, The Internal Architecture of the Femur and Its Clinical Significance: The Upper End, Journal of Bone and Joint Surgery, 37(1): 57-88, 1955

[14] Carter DR, Orr TE, Fyhrie D P, Relationships between loading history and femoral cancellous bone architecture, Journal of Biomechanics 22(3):231244,1989 .

[15] Jacobs CR, Simo JC, Beaupré GS, Carter DR, Adaptive bone remodeling incorporating simultaneous density and anisotropy considerations, Journal of Biomechanics 30(6):603-613, 1997.

[16] Cowin SC, The relationship between the elasticity tensor and the fabric tensor, Mechanics of Materials 4(2):137-147, 1985.

[17] Cowin SC, Wolff's Law of Trabecular Architecture at Remodeling Equilibrium, Journal of Biomechanical Engineering 108(1):83, 1986.

[18] Christen P, Ito K, Ellouz R, Boutroy S, Sornay-Rendu E, Chapurlat RD, Van Rietbergen B, Bone remodelling in humans is load-driven but not lazy, Nature Communications 5:1-5, 2014.

[19] Schulte FA, Ruffoni D, Lambers FM, Christen D, Webster DJ, Kuhn G, Müller R, Local Mechanical Stimuli Regulate Bone Formation and Resorption in Mice at the Tissue Level, PLoS ONE 8(4):e62172, 2013.

[20] Sugiyama T, Price JS, Lanyon LE, Functional adaptation to mechanical loading in both cortical and cancellous bone is controlled locally and is confined to the loaded bones, Bone 46(2):314-321, 2010.

[21] Huiskes R and Ruimerman R, van Lenthe GH, Janssen JD, Effects of mechanical forces on maintenance and adaptation of form in trabecular bone, Nature 405(6787):704-706, 2000.

[22] Ruimerman R, Van Rietbergen B, Hilbers P, Huiskes R, A 3-dimensional computer model to simulate trabecular bone metabolism, Biorheology 40(13):315-20, 2003. 
[23] Van Der Linden JC, Verhaar JAN, Weinans H, A Three-Dimensional Simulation of Age-Related Remodeling in Trabecular Bone, Journal of Bone and Mineral Research 16(4): doi = 10.1359/jbmr.2001.16.4.688, 688-696, 2001.

[24] Badilatti SD, Christen P, Levchuk A, Marangalou JH, van Rietbergen B, Parkinson I, Müller R, Large-scale microstructural simulation of loadadaptive bone remodeling in whole human vertebrae, Biomechanics and Modeling in Mechanobiology 15(1):83-95, 2016.

[25] Mullender MG, Huiskes R, Proposal for the Regulatory Mechanism of Wolff's Law, Journal of Orthopaedic Research 13(4):503-12, 1995.

[26] Van Oers RFM, Ruimerman R, Tanck E, Hilbers PAJ, Huiskes R, A unified theory for osteonal and hemi-osteonal remodeling, Bone 42(2):250-259, 2008.

[27] Van Oers RFM, Van Rietbergen B, Ito K, Hilbers PAJ, Huiskes R, A sclerostin-based theory for strain-induced bone formation, Biomechanics and Modeling in Mechanobiology 10(5):663-670, 2011.

[28] Tsubota KI, Adachi T, Tomita Y, Functional adaptation of cancellous bone in human proximal femur predicted by trabecular surface remodeling simulation toward uniform stress state, Journal of Biomechanics 35(12):15411551, 2002.

[29] Langton CM, Haire TJ, Ganney PS, Dobson CA, Fagan MJ, Dynamic Stochastic Simulation of Cancellous Bone Resorption, Bone 22(4):375380, 1998.

[30] Thomsen JS, Mosekilde L, Boyce RW, Mosekilde E, Stochastic simulation of vertebral trabecular bone remodeling, Bone 15(6):655-666, 1994.

[31] Rovati M, Taliercio A, Optimal Orientation of the Symmetry Axes of Orthotropic 3-D Materials, in Eschenauer, HA, Mattheck C, Olhoff N (eds.), Engineering Optimization in Design Processes, pp. 127-134, 1991.

[32] Banichuk NV, Optimization of Anisotropic Properties for Continuum Bodies and Structural Elements Using Spectral Methods of Tensor Analysis, Mechanics of Structures and Machines 24(1):71-87, 1996.

[33] Pedersen P, On optimal orientation of orthotropic materials, Structural Optimization 1(2):101-106, 1989.

[34] Cowin SC, Optimization of the strain energy density in linear anisotropic elasticity, Journal of Elasticity 34(1):45-68, 1994.

[35] Cowin SC, On the minimization and maximization of the strain energy density in cortical bone tissue, Journal of Biomechanics 28(4):445-447, 1995. 
[36] Fernandes P, Rodrigues H, Jacobs CR, A Model of Bone Adaptation Using a Global Optimisation Criterion Based on the Trajectorial Theory of Wolff, Computer Methods in Biomechanics and Biomedical Engineering 2(2):125138, 1999.

[37] Fyhrie DP, Carter DR, A unifying principle relating stress to trabecular bone morphology, Journal of Orthopaedic Research 4(3):304:317, 1986.

[38] Fyhrie DP, Schaffler MB, The adaptation of bone apparent density to applied load, Journal of Biomechanics 28(2):135-146, 1995.

[39] Waffenschmidt T, Menzel A, Kuhl E, Anisotropic density growth of bone-A computational micro-sphere approach, International Journal of Solids and Structures, 49:1928-1946, 2012.

[40] Huiskes R, Weinans H, Grootenboer HJ, Dalstra M, Fudala B, Slooff TJ, Adaptive bone-remodeling theory applied to prosthetic-design analysis, Journal of Biomechanics 20(11-12):1135-1150, 1987.

[41] Miller Z, Fuchs MB, Arcan M, Trabecular bone adaptation with an orthotropic material model, Journal of Biomechanics 35(2):247-256, 2002.

[42] Pettermann HE, Reiter TJ, Rammerstorfer FG, Computational simulation of internal bone remodeling, Archives of Computational Methods in Engineering 4(4):295-323, 1997.

[43] Geraldes DM, Modenese L, Phillips ATM, Consideration of multiple load cases is critical in modelling orthotropic bone adaptation in the femur, Biomechanics and Modeling in Mechanobiology 15(5):1029-1042, 2016.

[44] Cowin SC, Sadegh AM, Luo GM, An Evolutionary Wolff's Law for Trabecular Architecture, Journal of Biomechanical Engineering 114(1):129, 1992.

[45] Doblaré M, García JM, Anisotropic bone remodelling model based on a continuum damage-repair theory, Journal of Biomechanics 35(1):1-17, 2002.

[46] Ramtani S, Zidi M, Damaged-bone remodeling theory: Thermodynamical approach, Mechanics Research Communications 26(6):701-708, 1999.

[47] DiCarlo A, Quiligotti S, Growth and balance, Mechanics Research Communications 29(6):449-456, 2002.

[48] Menzel A, Modelling of anisotropic growth in biological tissues, Biomechanics and Modeling in Mechanobiology 3(3):147-171, 2005.

[49] Reina JM, García-Aznar JM, Domínguez J, Doblaré M, Numerical estimation of bone density and elastic constants distribution in a human mandible, Journal of Biomechanics 40(4):828-836, 2007. 
[50] Goda I, Ganghoffer JF, Maurice G, Combined bone internal and external remodeling based on Eshelby stress, International Journal of Solids and Structures 94-95:138-157, 2016.

[51] Klika V, Pérez MA; García-Aznar JM; Maršík F; Doblaré M, A coupled mechano-biochemical model for bone adaptation, Journal of Mathematical Biology 69(6-7):1383-1429, 2014.

[52] Komarova SV, Mathematical model of paracrine interactions between osteoclasts and osteoblasts predicts anabolic action of parathyroid hormone on bone, Endocrinology 146(8):3589-3595, 2005.

[53] Lemaire V, Tobin FL, Greller LD, Cho CR, Suva LJ, Modeling the interactions between osteoblast and osteoclast activities in bone remodeling, Journal of Theoretical Biology 229(3):293-309, 2004.

[54] Maldonado S, Borchers S, Findeisen R, Allgöwer F, Mathematical modeling and analysis of force induced bone growth, Conference proceedings: ... Annual International Conference of the IEEE Engineering in Medicine and Biology Society. IEEE Engineering in Medicine and Biology Society. Annual Conference 1:3154-3157, 2006.

[55] Martínez-Reina J, García-Aznar JM, Domínguez J, Doblaré M, A bone remodelling model including the directional activity of BMUs, Biomechanics and Modeling in Mechanobiology 8(2):111-127, 2009.

[56] Pivonka P, Zimak J, Smith DW, Gardiner BS, Dunstan CR, Sims NA, Martin TJ, Mundy GR, Modèl structure and control of bone remodeling: A theoretical study, Bone 43(2):249-263, 2008.

[57] Germain P, The Method of Virtual Power in Continuum Mechanics. Part 2: Microstructure, SIAM Journal on Applied Mathematics 25(3):556-575, 1973.

[58] Al Nazer R, Lanovaz J, Kawalilak C, Johnston JD, Kontulainen S, Direct in vivo strain measurements in human bone-A systematic literature review, Journal of Biomechanics 45(1):27-40, 2012.

[59] Podio-Guidugli P, A Primer in Elasticity, Springer Netherlands, Dordrecht, 2000.

[60] Sansalone V, Naili S, Di Carlo A, On the rotary remodelling equilibrium of bone,Computer Methods in Biomechanics and Biomedical Engineering 14(sup1):203-204, 2011.

[61] Van Buskirk WC, Ashman R, The elastic moduli of bone, in Cowin SC (ed.), Mechanical Properties of Bone, American Society of Mechanical Engineers, Colorado, pp. 131-143, 1981. 
[62] Beaupré GS, Orr TE, Carter DR, An approach for time-dependent bone modeling and remodeling-theoretical development, Journal of Orthopaedic Research 5(8):651-661, 1990.

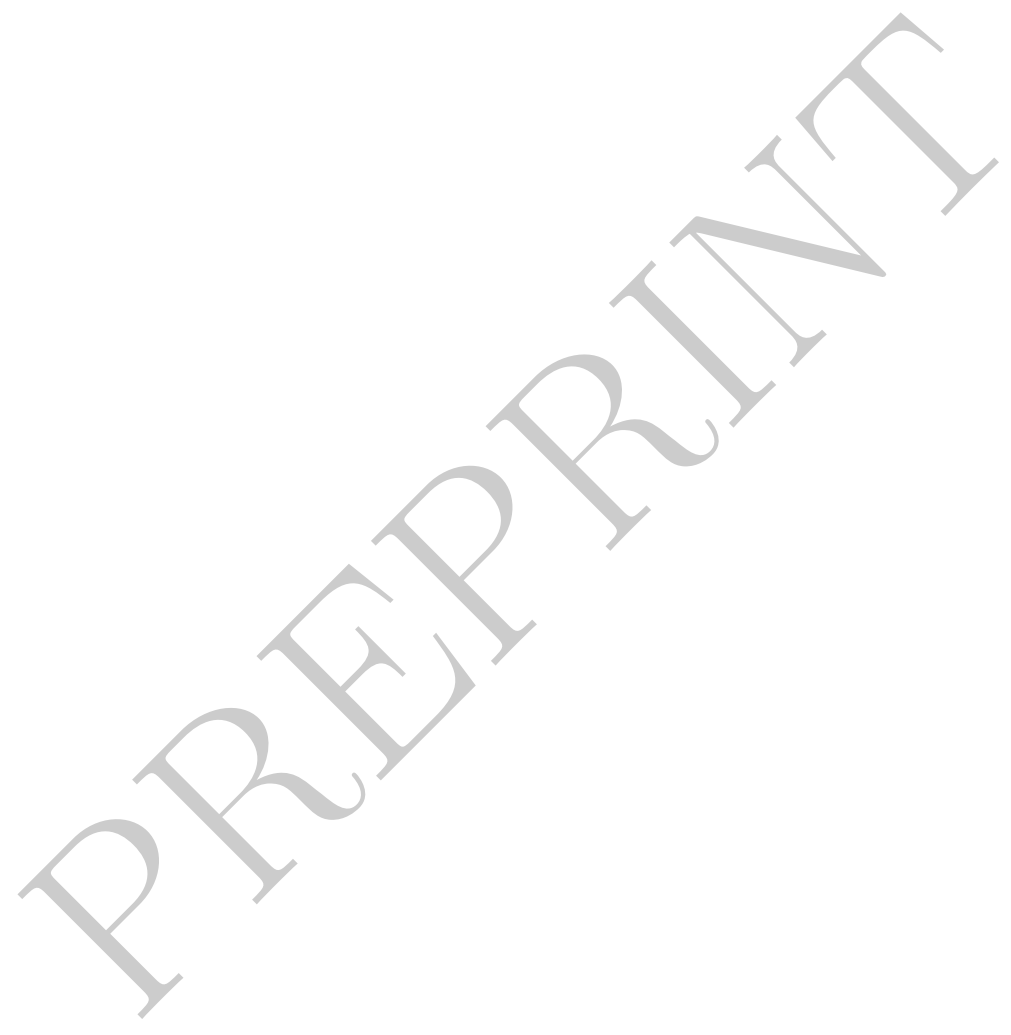



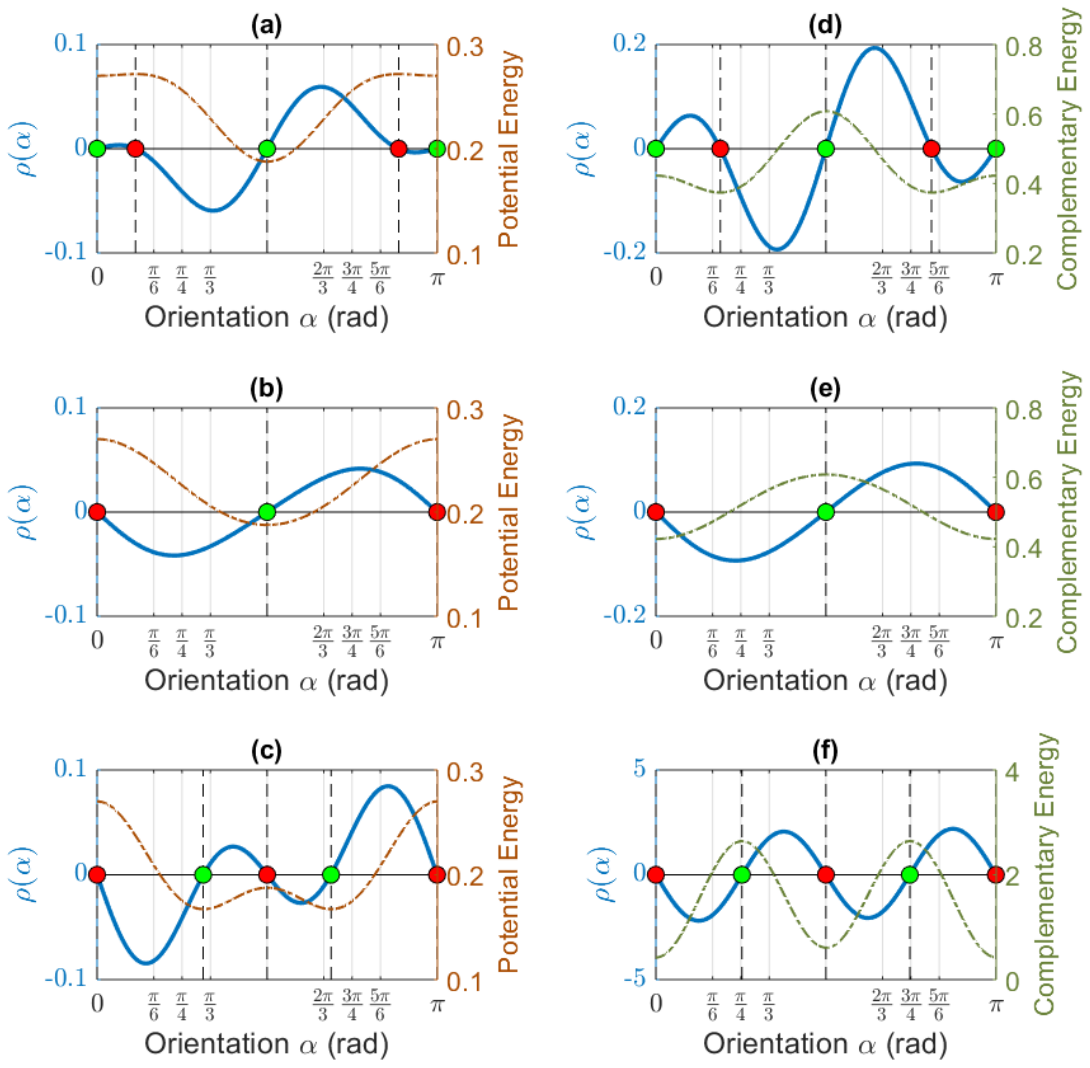

Figure 2: Rotary remodeling in uniaxial strain/stress conditions: functions $\rho$ (solid blue lines), strain energy $\psi^{\mathrm{E}}$ (brown dotted lines) and complementary energy $\psi^{\mathrm{S}}$ (dark green dotted lines) versus the angle $\alpha$ describing the orientation of material axes in the plane $\left(\mathbf{e}_{1}, \mathbf{e}_{2}\right)$. Subplots $(\mathrm{a}, \mathrm{b}, \mathrm{c})$ refer to uniaxial strain in the $\mathbf{e}_{1}$ direction $\left(E_{11}=3000 \mu \epsilon\right)$ and material types A (a), B (b) and C (c). Subplots (d,e,f) refer to uniaxial stress in the $\mathbf{e}_{1}$ direction $\left(S_{11}=100 \mathrm{MPa}\right)$ and material types A (d), B (e) and C (f). Stable and unstable equilibrium points are depicted by green and red knots, respectively. 

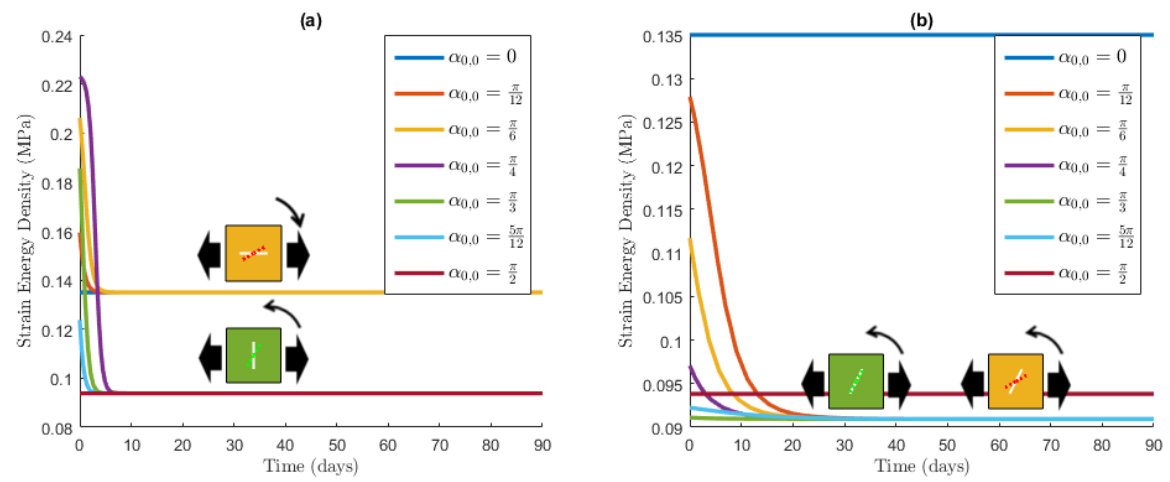

Figure 3: Evolution of the strain energy of a 2D body under uniform uniaxial strain, with varying initial states $\left\{\alpha_{0}=k \frac{\pi}{12}, k \in\{0,1,2,3,4,5,6\}\right\}$. (a) Material A (high shear modulus); (b) Material C (low shear modulus)

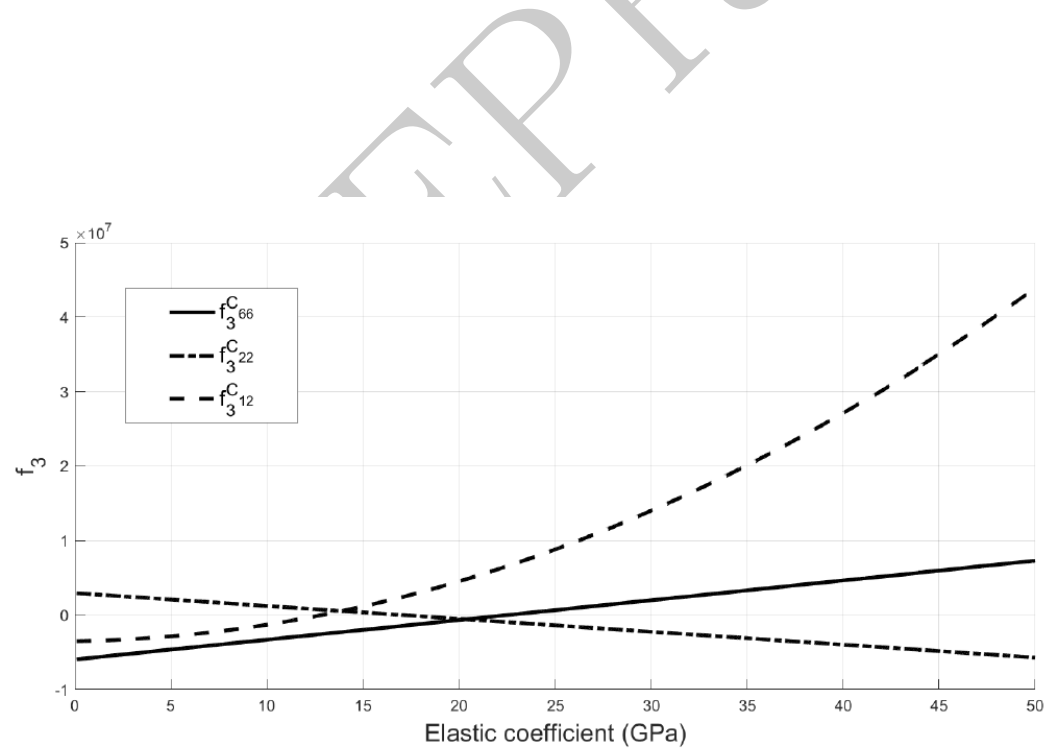

Figure 4: Variations of the function $f_{3}$ as a function of $C_{66}, C_{22}$ and $C_{12}$, where the reference material properties are type A (Table 1, high shear modulus). 
(a)

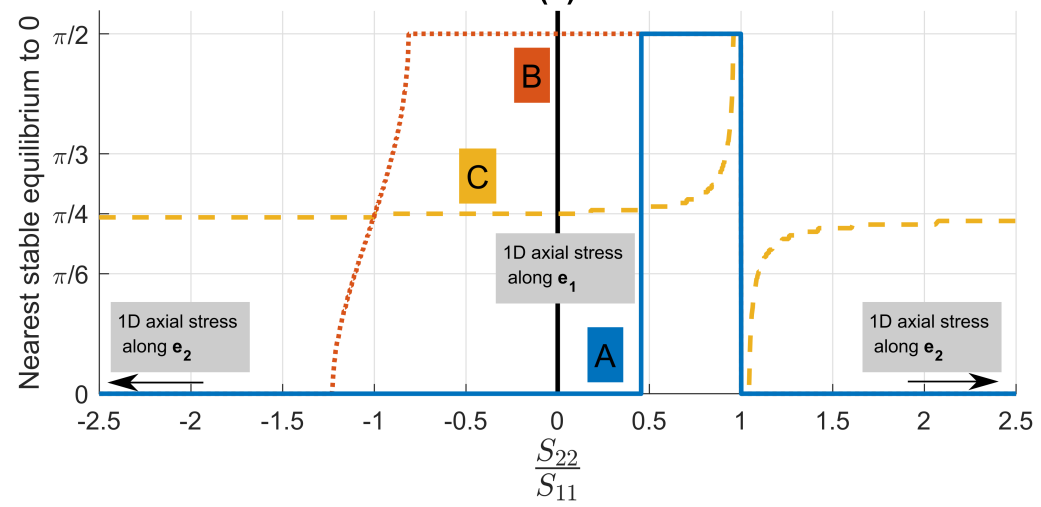

(b)

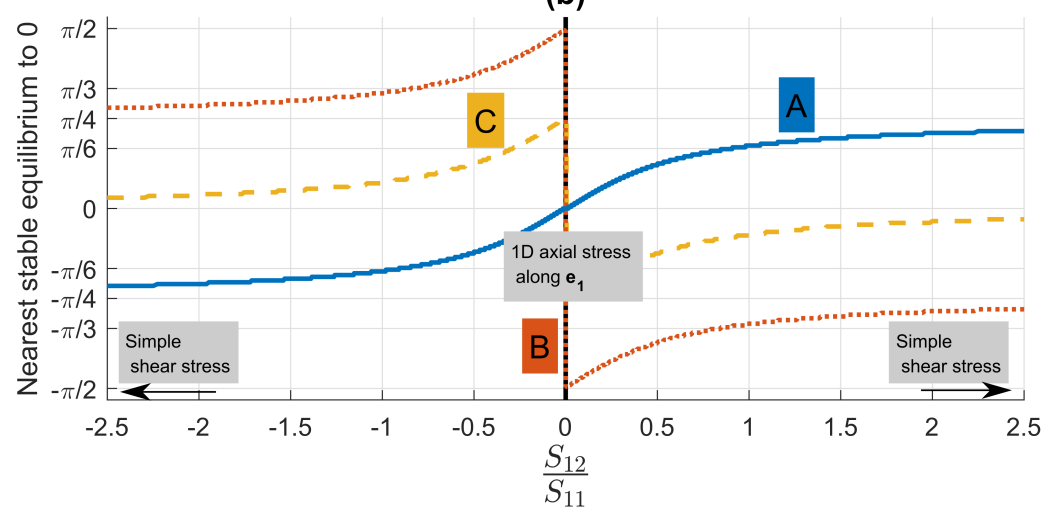

Figure 5: Closest stable equilibrium states to initial configuration $\alpha_{0}=0$, for varying (a) transverse/longitudinal stress ratio $\frac{S_{22}}{S_{11}}\left(S_{12}=0\right)$ and (b) shear/longitudinal stress ratio $\frac{S_{12}}{S_{11}}\left(S_{22}=0\right)$. The three curves account for the 3 material types in Table 1characterized by high (A, solid blue lines), mild (B, dotted dark-red lines), and low ( $\mathrm{C}$, dashed orange lines) shear moduli. 
(a)

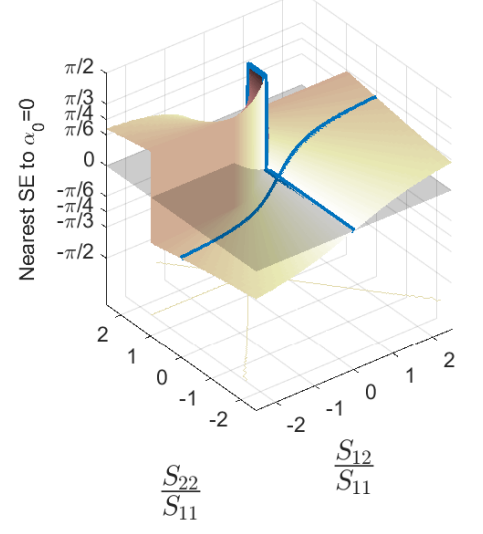

(c)

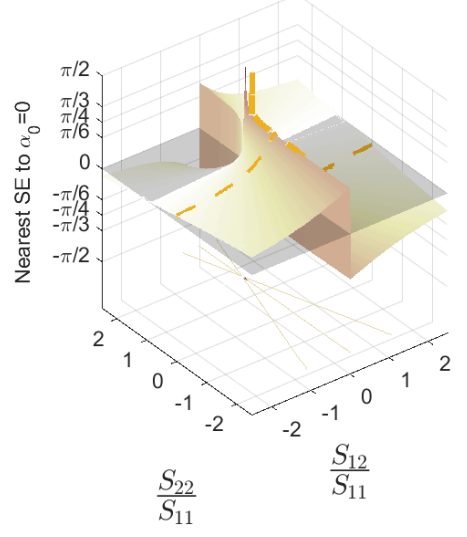

(b)
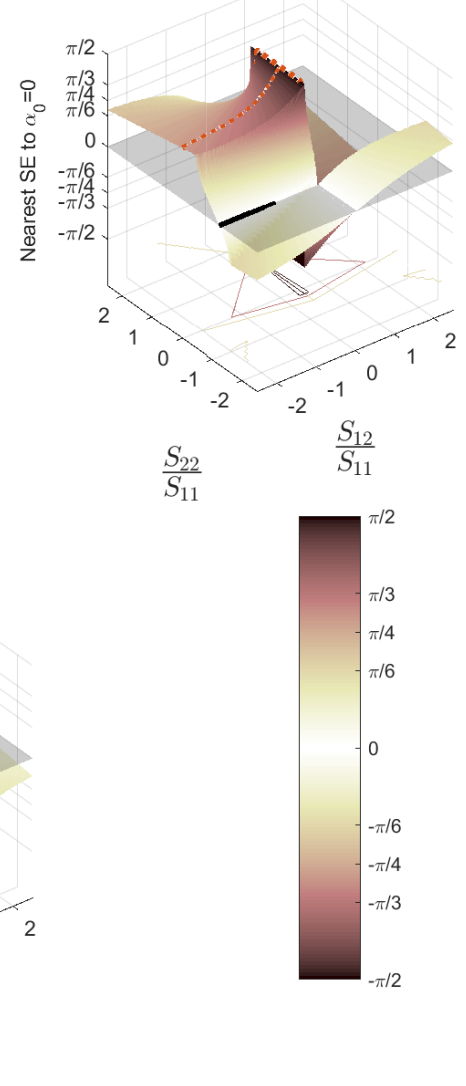

Figure 6: Closest stable equilibrium states to the initial configuration $\alpha_{0}=0$ as a function of transverse/longitudinal stress ratio $\frac{S_{22}}{S_{11}}$ and shear/longitudinal stress ratio $\frac{S_{12}}{S_{11}}$. The three plots (a,b,c) account for the 3 material types (A, B, C) in Table 1, respectively. Bold lines superimposed to the surfaces represent the lines in Fig. 5. 

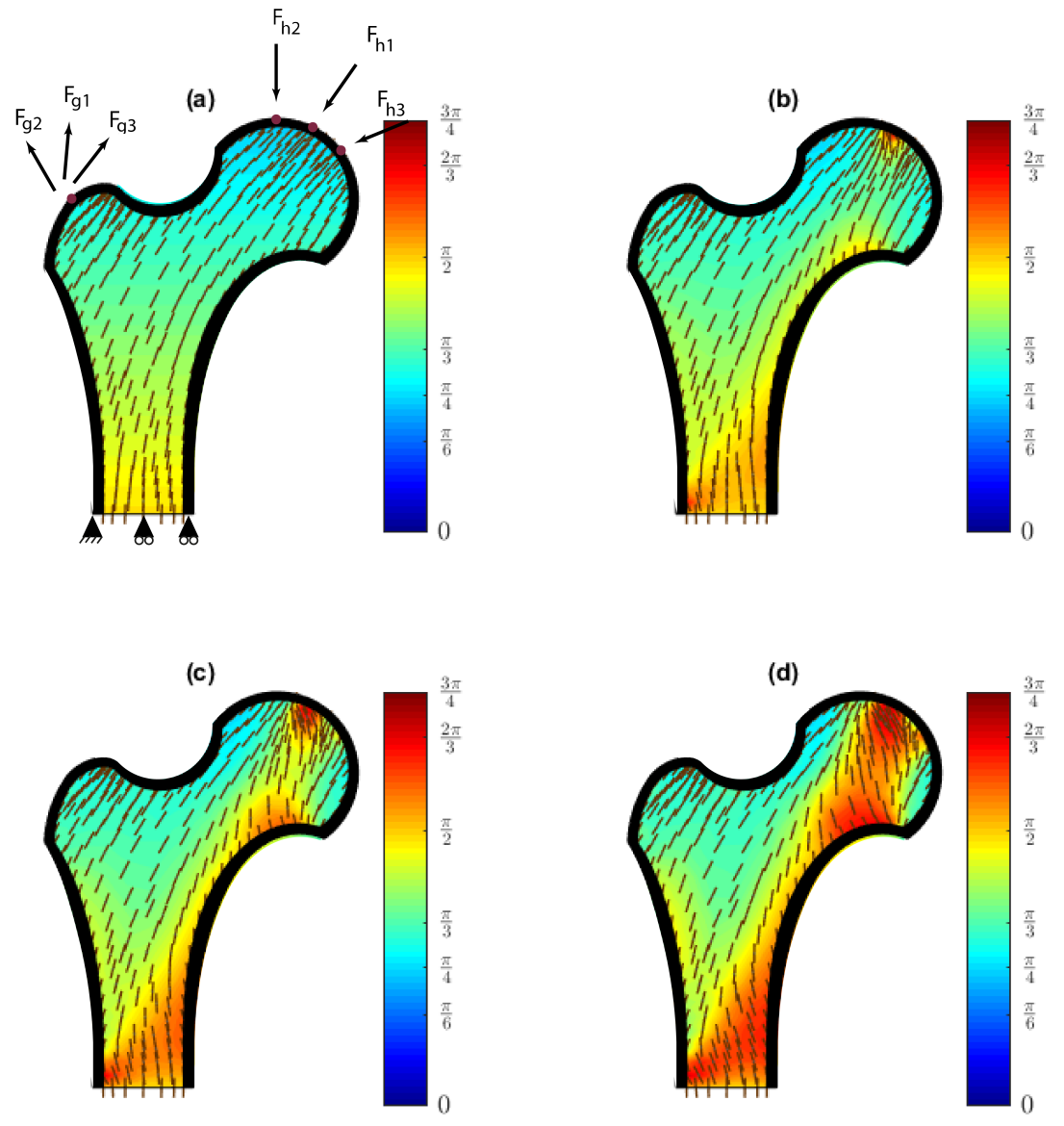

Figure 7: Evolution of the strain energy and micro-orientation during remodeling in a 2D toy model of the proximal femur: micro-orientation (brown sticks, and color map (rad)) at the start of the simulation, with loading and boundary conditions (a), after 500 (b), 1000 (c) and 2000 (d) time increments (arbitrary time scale). 